\title{
From bioactive pyrrolidino[3,4-c]pyrrolidines to more bioactive pyrrolidino[3,4-b]pyrrolidines via ring-opening ring-closing promoted by sodium methoxide
}

\author{
Samet Belveren, ${ }^{\mathrm{a}}$ Olatz Larrañaga, ${ }^{\mathrm{b}, \mathrm{c}}$ Samet Poyraz, ${ }^{\mathrm{a}} \mathrm{H}$.

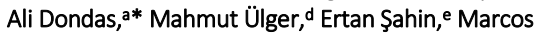 \\ Ferrándiz-Saperas, b,f José M. Sansano, b,f* M. de Gracia \\ Retamosa,, ,f and Abel de Cózar. $b, c, g *$ \\ ${ }^{\text {a }}$ Department of Chemistry. Faculty of Pharmacy, Mersin \\ University, 33169, Mersin, Turkey. \\ ${ }^{\mathrm{b}}$ Centro de Innovación en Química Avanzada (ORFEO-CINQA) \\ c Departamento de Química Orgánica I, Facultad de Química, \\ Universidad del País Vasco/Euskal Herriko Unibertsitatea \\ UPV/EHU, and Donostia International Physics Center (DIPC), P. \\ K. 1072, E-20018 San Sebastián, Spain \\ ${ }^{\mathrm{d}}$ Department of Pharmaceutical Microbiology. Faculty of \\ Pharmacy, Mersin University, 33169, Mersin, Turkey. \\ ${ }^{\mathrm{e}}$ Department of Chemistry. Faculty of Science and Arts, Atatürk \\ University, 25240 Erzurum, Turkey. \\ ${ }^{\mathrm{f}}$ Departamento de Química Orgánica, Instituto de Síntesis \\ Orgánica. Universidad de Alicante, Apdo. 99, E-03080-Alicante, \\ Spain. \\ ${ }^{g}$ IKERBASQUE, Basque Foundation for Science, 48011 Bilbao, \\ Spain. \\ *Corresponding Author for experimental: yakdas25@mersin.edu.tr, \\ jmsansano@ua.es \\ ¥Corresponding Author for calculations: abel.decozar@ehu.es
}

RING-OPENING-RING CLOSING-EPIMERIZATION

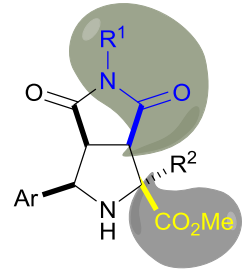

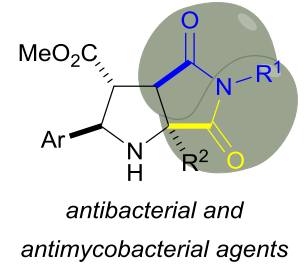

\section{Received:
Accepted:}

Publishec

Abstract The process involving a rearrangement of pyrrolidino[3,4c]pyrrolidine to another pyrrolidino[3,4-b]pyrrolidine involving sodium methoxide as base is fully studied. The effects of the substituents are analyzed during the ring-opening ring-closing sequence. Computational studies are also performed to explain the importance of susbstituents and quaternary carbons, especially when the (3-indolyl)methyl is present in the starting material. Finally, all the samples are evaluated as potential candidates as antibacterial and antimycobacterial agents.

Key words cycloaddition $\bullet$ azomethine ylides $\bullet$ rearrangement $\bullet$ antibacterials - DFT calculations

\section{Introduction}

The design of very simple molecular architectures with the broadest biological and medicinal coverage is always pursued, and especially for the long treatment of degenerative illnesses. A clear example is represented by succinimides. ${ }^{1}$ Activities such as CNS depressant, analgesic, antitumor, antispasmodic, bacteriostatic, hypotensive, antibacterial, antifungal, antitubercular, etc., have been reported in the literature.1,2,3 Succinimides are easily available from succinic acid or succinic anhydride and their derivatives involving ring-opening/ringclosing strategies. ${ }^{1,4}$ However, the imido group and the double bond of maleimides offer new substitution patterns. For example, their electrophilic character made them excellent dienophiles in Diels-Alder reactions and dipolarophiles in 1,3-dipolar cycloadditions. ${ }^{5}$ In fact maleimides are frequently used for the optimization of this cycloaddition processes.

During our investigation of the synthesis of new derivatives with a thiohydantoin framework ${ }^{6}$ (similar to 2) with antituberculosis and anti-bacterial activity, 7,8 we discovered the formation of unexpected compounds, which resulted from a rearrangement of the succinimide in the presence of sodium methoxide. The result of this rearrangement is a chemical switch in which from one fused succinimide with a tetrahydropyrrolo[3,4-c]pyrrole skeleton $\mathbf{1}$ it was possible access to a new succinimide with tetrahydropyrrolo[3,4b]pyrrole framework 3 (Scheme 1).

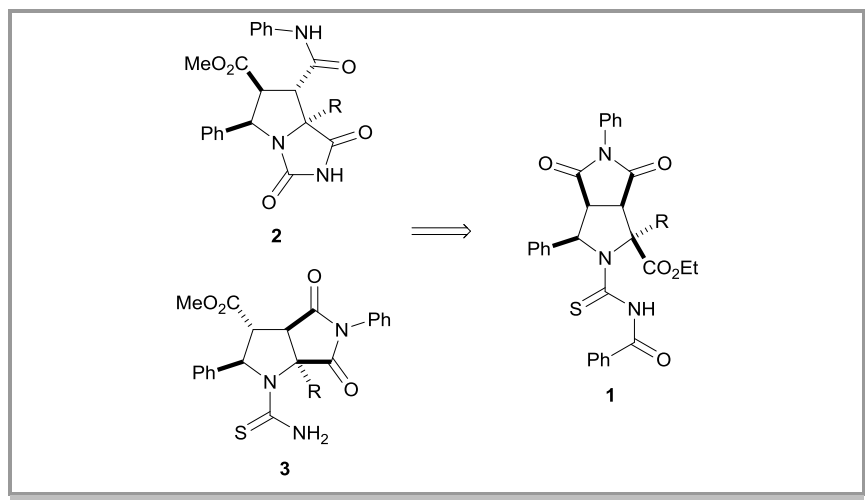

Scheme 1 First evidence of the titled succinimide rearrangement

In this work we thoroughly study the mechanism of the particular rearrangement originated by the methoxide anion, which attack to molecules 4 to give products 5 (Scheme 2). ${ }^{6} \mathrm{We}$ envisage the possible scope and its utility in synthetic organic chemistry and as antituberculosis and antibacterial agent. ${ }^{7}$

Results and Discusion 


\section{Scope of the rearrangement and structural determination of} compounds 5, 6 and 7.

Following the reaction conditions found in the confirmation of the structure of compound $\mathbf{5}\left(\mathrm{Ar}, \mathrm{R}^{1}=\mathrm{Ph}, \mathrm{R}^{2}=3\right.$-indolyl) in our previous publication, ${ }^{6}$ we started with the analysis of the tetrahydropyrrolo[3,4-c]pyrrole 4a obtained from 1,3-dipolar cycloaddition of the corresponding methyl benzylideneaminoglycinate with $\mathrm{N}$-methylmaleimide (NMM), (see experimental part). Under general conditions described in Scheme 2, compound $\mathbf{4 a}$ afforded a very complex mixture of unidentified products detected by ${ }^{1} \mathrm{H}$ NMR experiment of the crude reaction mixture.

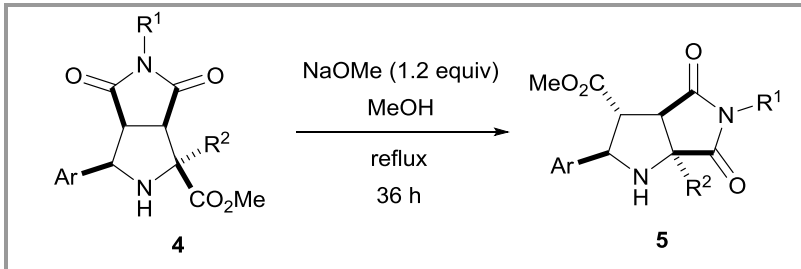

Scheme 2 Succinimide rearrangement studied in this work

Cycloadducts $\mathbf{4 b}-\mathbf{4 f}$, obtained from imino esters derived from leucine and phenylalanine were submitted to conditions depicted in Scheme 2, obtaining the corresponding tetrahydropyrrolo [3,4$b$ ]pyrroles 5b-f in moderate yield (up to 54\%, Figure 1). Despite purifying all these compounds in deactivated flash silica gel, we observed some decomposition/epimerization during this process. We also discovered that they were not stable under storage for more than one week at $-20^{\circ} \mathrm{C}$.

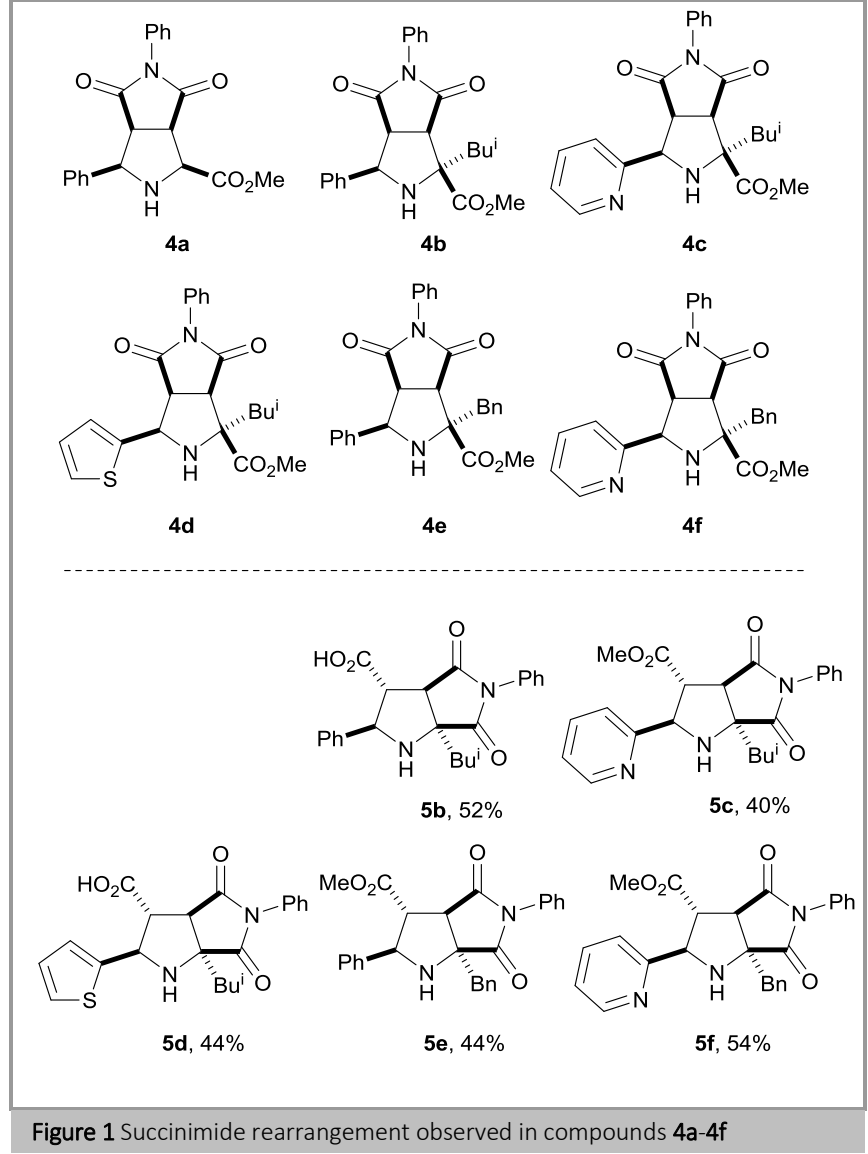

According to our experience, ${ }^{8}$ the introduction of an indol ring can be beneficial for increasing the biological effect of the substance. ${ }^{9}$ With this aim, cycloadducts $\mathbf{4 g}$, derived from tryptophan, were prepared (see experimental part) and were allowed to undergo the titled stereospecific rearrangement. Again, the reaction proceeded regio- and stereospecifically to give the corresponding compounds $\mathbf{5} \mathbf{g}^{10}$ in very high yields (70$98 \%$ ) (Scheme 3). These series of molecules $\mathbf{5 g}$ are very stable and could be stored for a long time.

The preparation of $\mathrm{N}$-benzoylcarbothioamides $\mathbf{6 g}$ was achieved smoothly by reaction of $\mathbf{5 g}$ with benzoylisothiocyanate in acetonitrile at room temperature for 24-30 h (Scheme 3). The incorporation of this unit to the pyrrolidine ring increases the biological potency of the precursor heterocycles. 


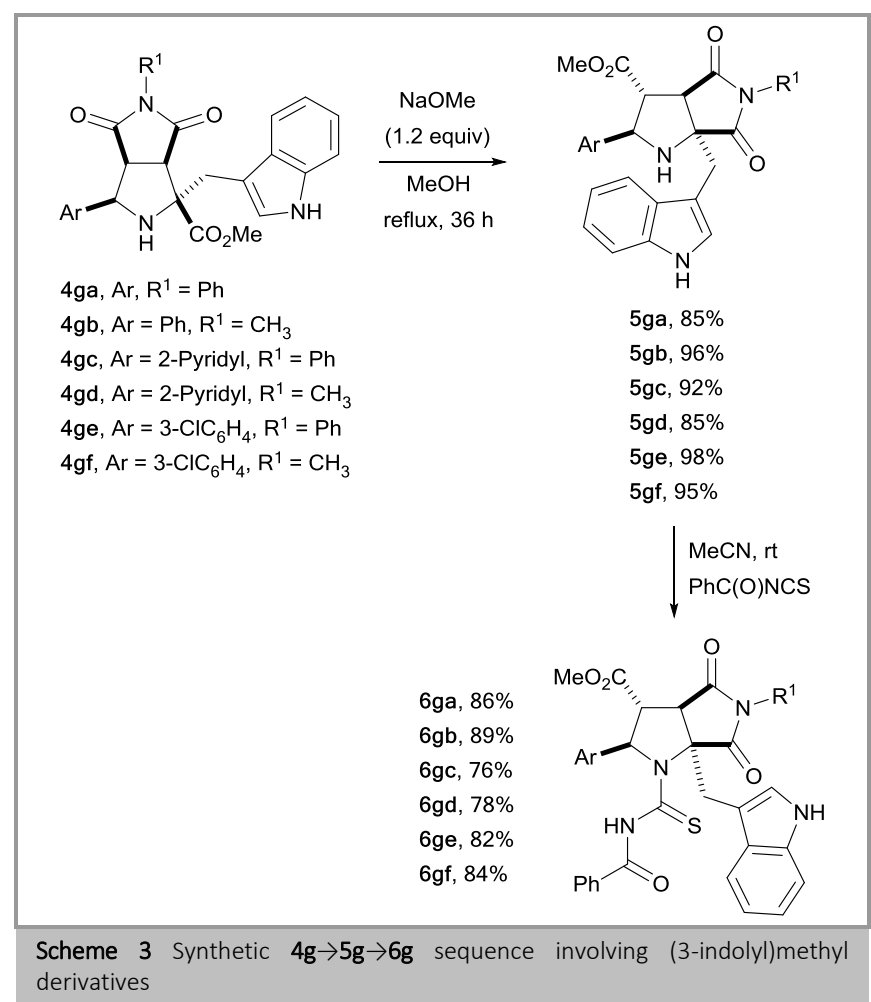

The relative configuration of all new racemic compounds was stablished according to data acquired using NMR experiments and by single crystal X-ray diffraction analysis for the compound 6gf (Figure 2).

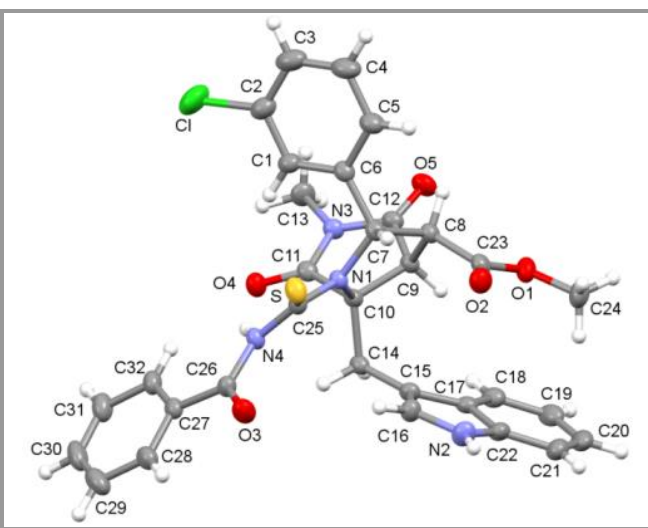

Figure $2 \mathrm{X}$-Ray diffraction analysis of compound $6 \mathrm{gf}$. Thermal ellipsoids are drawn at $40 \%$ probability level. ${ }^{11}$

A larger excess of sodium methoxide in methanol (not dry) furnished the same arrangement (under identical reaction conditions) afforded free betaproline amino acid ${ }^{12}$ derivative (possessing a zwitterionic structure) 7gg in almost quantitative yield (Scheme 4). The structure of its skeleton was also confirmed by X-ray diffraction analysis demonstrating that epimerization occurred only in the carbon atom 4 .

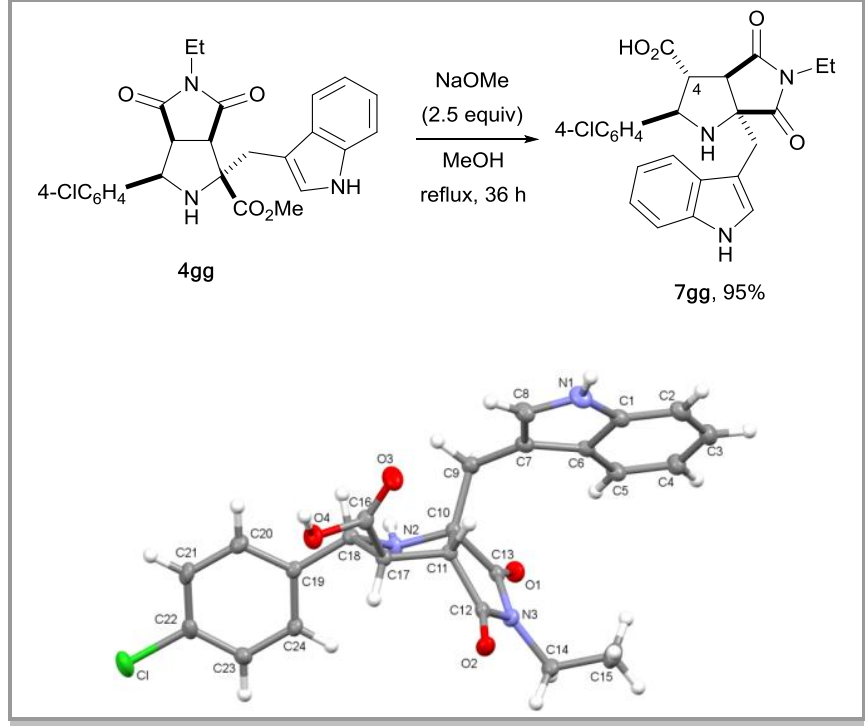

Scheme 4 Sythesis of betaproline derivative $7 g g$ and its X-ray diffraction analysis pattern. ${ }^{13}$

\section{Study of the mechanism by DFT calculations.}

At this point we can argue that the presence of a quaternary carbon at 2-position in the prolinate ring of compounds $\mathbf{4}$ seems to be crucial for the development of the arrangement in basic media. The Thorpe-Ingold effect can justify the scarce reactivity of cycloadduct $\mathbf{4 a}$ and the moderate to excellent yields achieved in substrates $\mathbf{4 b}$-g. Additionally, the presence of the (3indolyl)methyl residue at this position accelerated the process and gave an extra stability to the final compounds. We decided to perform computational calculations within the DFT framework in order to better understand the reaction mechanism associated with succinimide $\mathbf{4}$ rearrangement and its subsequent isomerization to yield compounds 5. For that, we selected 4ga as model compound. In the first part of this study we analyzed all the possible reactions of methoxide anion with 4ga. This anion can act as a nucleophile, reacting with the CO double bond of the imido groups (TS1 and TS1' in Figure 4). On the other hand, methoxide can also act as base, therefore the abstraction the protons in $\alpha$ position of the imido groups of maleimide moiety were also considered (Figure 3). 


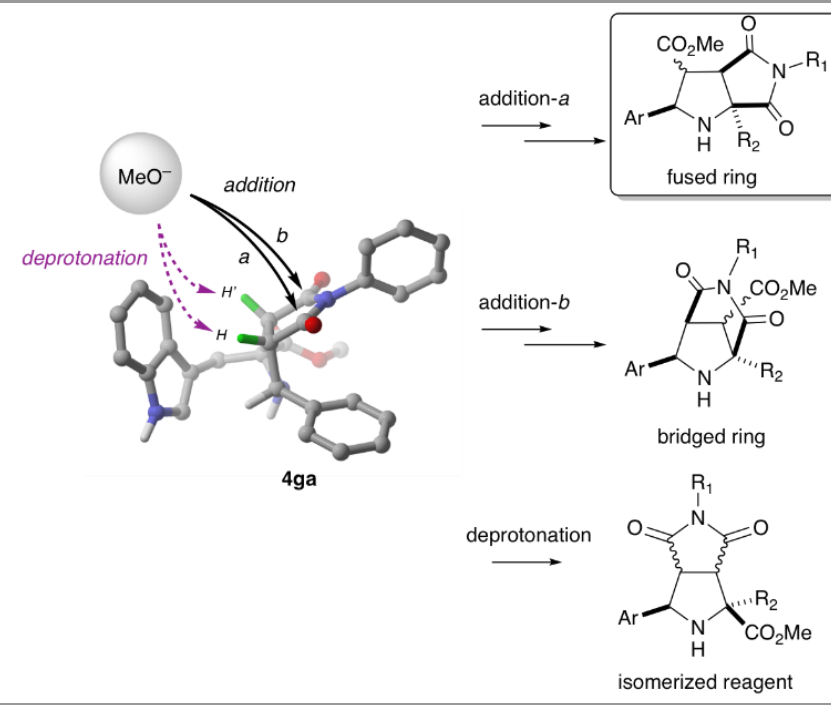

Figure 3 Possible reactions of compound $4 \mathrm{ga}$ with methoxide anion. Acidic hydrogens considered are highlighted in green

The main geometrical features of the transition structures associated with these processes and their relative energies are collected in Figure 4.

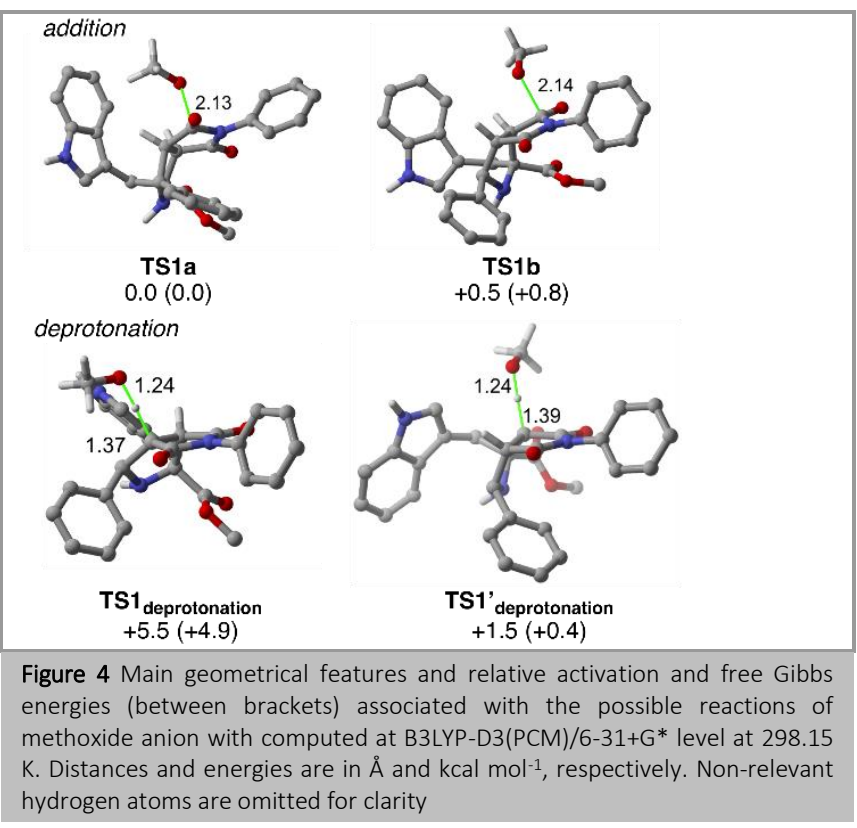

Our calculations shown that the activation energy barriers associated with the methoxide addition are lower than the deprotonation ones. This difference is even smaller considering Gibbs free activation barriers. However, the corresponding enolates formed are high in energy, consequently, their formation is thermodynamically disfavored. Therefore, any possible isomerization of compound 4ga via direct proton abstraction will not be further considered in this study. In addition, calculations also show that bridged ring formation is also kinetically disfavored (See supporting information for further details about other possible reaction paths computationally analyzed). Within these results, we next analyzed the succinimide rearrangement processes leading towards formation of fused rings $\mathbf{5}$. The relative and activation energies (and Gibbs free energies) computed are collected in Scheme 5. The main geometrical features of the corresponding transition structures are depicted in Figure 5.

$$
\text { (N) }
$$

Scheme 5 Activation and relative energies (and Gibbs free energies between brackets) associated with 4ga rearrangement with methoxide anion computed at B3LYP-D3 / 6-31+G(d) level of theory at $298 \mathrm{~K}$. Energies are in $\mathrm{kcal} \mathrm{mol}^{-1}$

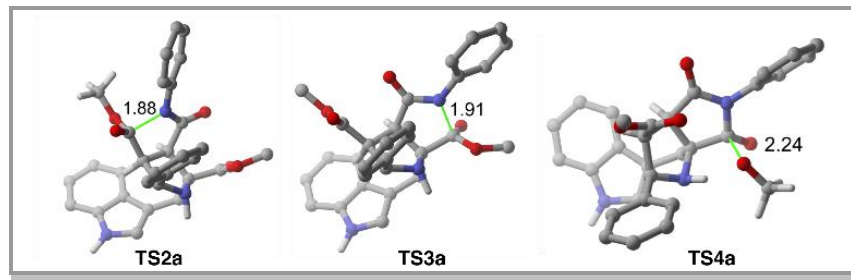

Figure 5 Main geometrical features and relative activation and free Gibbs' energies (between brackets) associated with 4ga rearrangement. See caption of Figure 3 for further details

Within the proposed mechanism, formation of the new maleimide ring is the rate-limiting step (TS3a has activation barrier ca. $1 \mathrm{kcal} \mathrm{mol}^{-1}$ higher than any other step). Moreover, calculations show that formation of INT4a is thermodynamically disfavored.

Once formation of INT4a via ring-opening ring-closing mechanism was assessed, we next analyzed computationally the subsequent isomerization towards ring-fused 5ag. Relative and activation energies (and Gibbs free energies) and main geometrical features of the corresponding transition structures are collected in Scheme 6. 


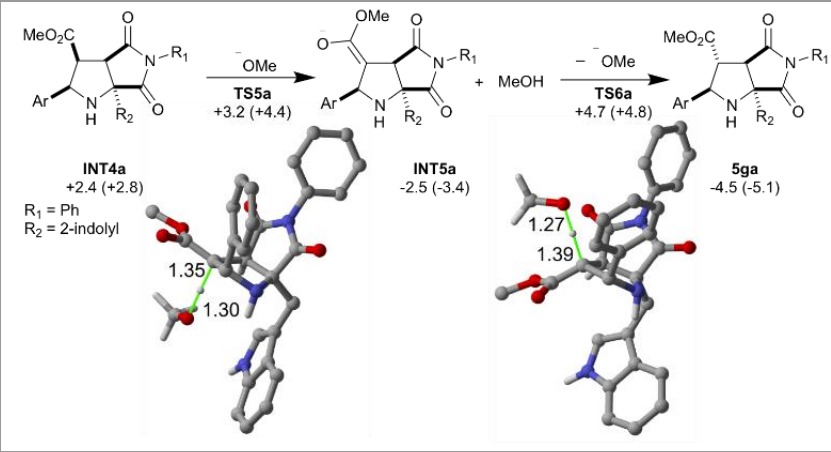

Scheme 6 Activation and relative energies (and Gibbs free energies between brackets) associated with 5 ga formation. See caption of Figure 3 for further details

Our calculations indicate that the isomerization of INT4a towards 5ga formation is thermodynamically favored, as reflected by its stability. Geometry inspection revealed that INT4a is highly energetic structural arrangement due to the repulsion associated with the eclipsed conformation of methoxycarbonyl and maleimide moieties. That repulsion is dismissed due to the isomerization process, being replaced by a stabilizing $\pi, \pi$-stacking interaction with the 3-indolyl moiety, and a close indole-ester hydrogen bonding, thus making this step the driving force of the reaction (Figure 6). Remarkably, the activation barriers associated with the proposed mechanism are lower than $6 \mathrm{kcal} \mathrm{mol}^{-1}$, compatible with the relatively mild conditions experimentally required (reaction temperature of 65 $\left.{ }^{\circ} \mathrm{C}\right)$. These stabilizing interactions, which did not exist in compounds 5b-f, can be the reasons of the epimerization/decomposition of these last molecules.

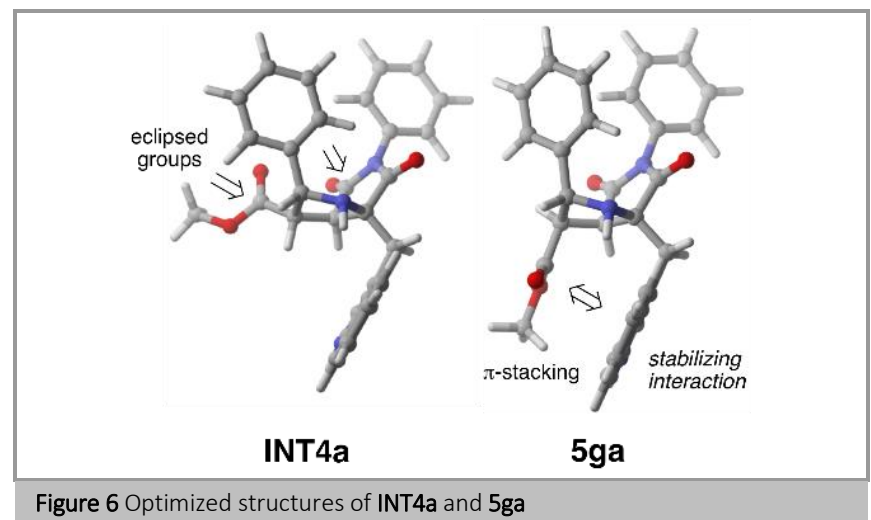

Figure 6 Optimized structures of INT4a and 5ga

\section{Anti-mycobacterial Activity.}

Anti-mycobacterial activity of prepared compounds were tested against M. tuberculosis H37Rv strain using Microplate Alamar Blue assay according to literature method ${ }^{14}$ measured by means of MIC values $(\mu \mathrm{g} / \mathrm{mL}$ ). Ethambutol (EMB) (Sigma E4630) and isoniazid (INH) (Sigma I3377) were used as standard reference drugs. The Anti-TB activity against M. tuberculosis H37Rv strain showed moderate activity, in the range of 10$80 \mu \mathrm{g} / \mathrm{mL}$, when compared to isoniazid and ethambutol as known reference drugs (Table 1). Especially the compounds 4 gf (possess - $\mathrm{CI}$ on the phenyl ring and -Me on the melaimide ring) revealed the highest activities with the MIC values of $10 \mu \mathrm{g} / \mathrm{mL}$ whereas the compound 4ga, 4gc, 4ge, 6gd and 7 gg showed activity in value of $20-40 \mu \mathrm{g} / \mathrm{mL}$ and the others compounds showed the lowest activities with the MIC values of $80 \mu \mathrm{g} / \mathrm{mL}$. In addition the tested compounds exhibited better anti-TB activity when compared their anti-bacterial activity as indicated in Table 1. Although the mode of action or biological target of these molecules is unknown at the moment, further work to get more potent derivatives is under investigation.

\section{Anti-bacterial Activity.}

Antibacterial activity of prepared compounds were tested against two Gram(+) bacteria Staphylococcus aureus (ATCC 25925), Bacillus subtilis (ATCC 6633) and three Gram(-) bacteria Escherichia coli (ATCC 25923), Acinetobacter baumannii (ATCC 02026), Aeromonas hydrophila (ATCC 95080) which obtained from the Refik Saydam Hifzısıhha Institute, Ankara, Turkey. Ampicillin was used as control drug. The minimum inhibitory concentrations (MIC) values was determined by agar dilution in duplicate as recommended by the Clinical Laboratory Standards Institute. ${ }^{15}$ To ensure that the solvents had no effect on microbial growth, a control test was performed containing inoculated broth supplemented with DMSO at the same dilutions used for the test compounds and was determined to be inactive.

The tested compounds inhibited the growth of bacteria at MIC values in the range of $62.5-500 \mu \mathrm{g} / \mathrm{mL}$ whereas the control, ampicillin, showed activity against the tested bacterla with a range of $125-0.9 \mu \mathrm{g} / \mathrm{mL}$ as given in Table 1 . It is also important to note that the screened compounds were found to show the better activity against A.baumannii (ATCC 02026) in the range of 62.5$125 \mu \mathrm{g} / \mathrm{mL}$ whereas the control ampicillin showed activity in MIC values of $125 \mu \mathrm{g} / \mathrm{mL}$.

Table 1. The MIC values $(\mu \mathrm{g} / \mathrm{mL})$ of the tested compounds against the bacterial and mycobacterial strains.

\begin{tabular}{|c|c|c|c|c|c|c|}
\hline & $\begin{array}{l}\text { S. aureus (ATCC } \\
25925 \text { ) }\end{array}$ & $\begin{array}{l}\text { E. coli (ATCC } \\
25923)\end{array}$ & $\begin{array}{l}\text { A. baumannii (ATCC } \\
\text { 02026) }\end{array}$ & $\begin{array}{lr}\text { B. } & \text { subtilis } \\
\text { (ATCC 6633) }\end{array}$ & $\begin{array}{l}\text { A. hydrophila (ATCC } \\
95080 \text { ) }\end{array}$ & $\begin{array}{l}\text { M. tuberculosis } \\
\text { H37Rv }\end{array}$ \\
\hline 4ga & 250 & 125 & 62,5 & 125 & 125 & 20 \\
\hline 4gb & 125 & 125 & 125 & 125 & 125 & 80 \\
\hline $4 \mathrm{gc}$ & 125 & 125 & 62,5 & 125 & 125 & 40 \\
\hline 4gd & 250 & 125 & 62,5 & 125 & 125 & 80 \\
\hline 4ge & 125 & 125 & 62,5 & 125 & 125 & 40 \\
\hline
\end{tabular}




\begin{tabular}{|c|c|c|c|c|c|c|}
\hline $4 \mathrm{gf}$ & 125 & 125 & 62,5 & 62,5 & 62,5 & 10 \\
\hline $5 g d$ & 125 & 125 & 62,5 & 125 & 62,5 & 80 \\
\hline 6ga & 125 & 125 & 125 & 125 & 125 & 80 \\
\hline $6 \mathrm{gb}$ & 250 & 250 & 125 & 250 & 250 & 80 \\
\hline 6gd & 250 & 250 & 125 & 250 & 500 & 31.25 \\
\hline 6ge & 125 & 125 & 125 & 125 & 125 & 80 \\
\hline $6 \mathrm{gf}$ & 125 & 250 & 125 & 125 & 125 & 80 \\
\hline $7 \mathrm{gg}$ & 62,5 & 125 & 62,5 & 125 & 62,5 & 40 \\
\hline Ampicillin & 31.25 & 15.62 & 125 & 0.9 & 31.25 & \\
\hline Isoniazid & & & & & & 0.2 and 0.1 \\
\hline Etambuol & & & & & & 5 and 10 \\
\hline
\end{tabular}

\section{Conclusions}

The rearrangement of tetrahydropyrrolo[3,4-c]pyrrole skeleton to a new tetrahydropyrrolo[3,4-b]pyrrole structure could be efficiently controlled in basic media. The presence of quaternary carbons in the starting bicyclic succinimide favored the rearrangement. The presence of the (3-indolyl)methyl group attached to this quaternary carbon is crucial for the stability of the final rearranged succinimides, increasing the biological activity of this family of compounds. Calculation predictions were in agreement with the experimental findings: first, the methoxide anion attacked the carbonyl group rather than promote the deprotonation; second, the spontaneous isomerization afforded a much more stable compound; third, a stabilizing $\pi$-stacking interaction between the indole ring and the ester group bonded to the epimerized carbon atom was the driving force of the reaction. Compound $\mathbf{4 g f}$ was the most active compound after the evaluation of all biological tests.

\section{Experimental Section}

The commercially available reagents for syntheses and analyses were obtained with analytical grade and used as received. Column chromatography was performed on silica gel 60 (Merck, 230-400 mesh). Melting points were determined with a Reichert Thermovar hot plate apparatus and are uncorrected. Mass spectra were obtained using a Bruker AC-300 or AC-400, and were recorded at 300 or $400 \mathrm{MHz}$ for ${ }^{1} \mathrm{H}$ NMR and 75 or 100 $\mathrm{MHz}$ for ${ }^{13} \mathrm{C}$ NMR using $\mathrm{CDCl}_{3}$ and $\mathrm{MeOD}$ as a solvent. Chemical shifts are given in parts per million ( $\delta$ :) downfield from tetramethylsilane. The following abbreviations are used: $s$ singlet; $\mathrm{d}$ - doublet; $\mathrm{t}$ - triplet; $\mathrm{q}$ - quartet; $\mathrm{m}$ - multiplet; $\mathrm{br}$ broad. IR spectra were taken on a Perkin-Elmer Spectrum One FT-IR spectrometer and also were taken on Nicolet 510 P-FT. Low-resolution electron impact (EI) mass spectra were obtained at $70 \mathrm{eV}$ using a Shimadzu QP-5000 by injection or DIP; fragment ions in $\mathrm{m} / \mathrm{z}$ are given with relative intensities (\%) in parentheses. High-resolution mass spectra (HRMS) were measured on an instrument using a quadrupole time-of-flight mass spectrometer (QTOF) and also through the electron impact mode (EI) at $70 \mathrm{eV}$ using a Finnigan VG Platform or a Finnigan MAT 95S.The compounds are named according to the IUPAC system; names were obtained using MDL Autonom.

The known pyrrolidine derivative methyl $(1 \mathrm{~S}, 3 \mathrm{R})-1-((1 \mathrm{H}-$ indol-3-yl)methyl)-4,6-dioxo-3,5diphenyloctahydropyrrolo[3,4-c]pyrrole-1-carboxylate

Template for SYNTHESIS (C) Thieme, Stuttgart · New York and and aminocarbothiol pyrrolidine derivatives $(2 S, 3 S, 3 \mathrm{a} S, 6 \mathrm{a} R)$-Methyl $\quad 6$ a-( $(1 H$-indol-3-yl)methyl)octahydro4,6-dioxo-2,5-diphenylpyrrolo[ 3,4-b]pyrrole-3-carboxylate (5ga) were prepared as from literature. 16,6

Novel bicyclic pyrrolidine derivatives $\mathbf{4 g b - 4 g f}$ were prepared by modification of literature methods. ${ }^{17,6}$

\section{Computational Methods}

Theoretical calculations have been carried out at the B3LYPD3/6-31+G(D) ${ }^{18}$ level by using the GAUSSIAN $09^{19}$ suite of programs. Activation and relative (Gibbs) energies were computed within the DFT framework ${ }^{20}$ at the B3LYP-D3/6$31+G(D)$ level at $298 \mathrm{~K}$ in which dispersion corrections are included by means of Grimme's D3 model. ${ }^{21}$ Solvent effects were estimated by the polarization continuum model 22 (PCM) method within the self-consistent reaction field (SCRF) approach. ${ }^{23}$ All SCRF-PCM calculations were performed using dimethylsulfoxide ( $\varepsilon=46.826$ ) as model solvent. Merz-Kollman atomic radii cavities (as invoked by the radii= Pauling keyword) were used in reaction steps associated with hydrogen atom migration.

All the stationary points were characterized by harmonic vibrational analysis. Local minima showed positive definite Hessians. Fully optimized transition structures (TSs) showed one and only one imaginary frequency associated with nuclear motion along the chemical transformation under study. Reaction paths were checked by Intrinsic Reaction Coordinate (IRC) calculations. In order to avoid errors associated with $1 \mathrm{~N}$ solvation state, activation barriers were compute comparing energies of directly connected stationary points.

\section{General procedure for Preparation of pyrrolidines}

To a solution of the silver salt $(\mathrm{AgOAc})$ in toluene $(3 \mathrm{~mL})$ was added a solution of imino ester ( $1 \mathrm{mmol}$ ) and $N$-phenylmaleimide (1 mmol) in toluene $(2 \mathrm{~mL})$. To the resulting suspension trimethylamine $(0.05 \mathrm{mmol}, 7 \mu \mathrm{L})$ was added and the mixture stirred at room temperature $\left(20-30{ }^{\circ} \mathrm{C}\right)$ for $18-24 \mathrm{~h}$. The crude reaction mixture was filtered through a small Celite path. The residue was purified by flash chromatography or the solid products were recrystallized in mixture of $n$-hexane/ether. 
General procedure for rearrangement access to pyrrole-4,6diones

To a stirred solution of bicyclic pyrrolidine $\mathbf{4 a - 4 f}(1 \mathrm{mmol})$ in dry methanol $(10 \mathrm{~mL})$ was added dropwise a solution of sodium methoxide (1.2 mmol) in dry methanol $(10 \mathrm{~mL})$ over 10-15 min, and the mixture stirred and refluxed for $32-36 \mathrm{~h}$. The solvent was evaporated under reduced pressure and quenched with saturated aqueous ammonium chloride, then extracted with dichloromethane $(3 \times 15 \mathrm{~mL})$. The combined organic phases were dried over $\mathrm{MgSO}_{4}$ and filtered. The product $\mathbf{5 b - 5 f}$ were purified by flash chromatography, the silica gel was deactivated (a $5 \%$ of triethylamine was added as co-eluent) to improve the yield of the final product.

Methyl

(1S,3R,3aS,6aR)-4,6-dioxo-3,5diphenyloctahydropyrrolo[3,4-c]pyrrole-1-carboxylate

(4a): After 18 hours and work up the product was isolated as a white solid (318 mg, $91 \%$ yield); column chromatography ( $n$ hexane:EtOAc; 8:2); All spectra were in agreement with reported data.

\section{Methyl (1S,3R,3aS,6aR)-1-isobutyl-4,6-dioxo-3,5-} diphenyloctahydropyrrolo[3,4-c]pyrrole-1-carboxylate

(4b): After 18 hours and work up the product was isolated as a white solid (321 mg, $79 \%$ yield); column chromatography ( $n$ hexane:EtOAc; 8:2); mp: 145-149 으; IR (ATR) $v_{\max }: 1713,1502$, $1375,1206,1166,1140,854,702,692 \mathrm{~cm}^{-1}$; ${ }^{1} \mathrm{H}$ NMR $(300 \mathrm{MHz}$, $\left.\mathrm{CDCl}_{3}\right): \delta 0.89(\mathrm{~d}, J=6.5 \mathrm{~Hz}, 3 \mathrm{H}), 1.01(\mathrm{~d}, J=6.5 \mathrm{~Hz}, 3 \mathrm{H}), 1.69-1.87$ (m, 2H), 2.01-2.19 (m, 1H), $2.81(\mathrm{~d}, J=7.2 \mathrm{~Hz}, 1 \mathrm{H}, \mathrm{NH}), 3.38(\mathrm{~d}, J$ $=7.6 \mathrm{~Hz}, 1 \mathrm{H}), 3.76(\mathrm{dd}, J=9.3,7.6 \mathrm{~Hz}, 1 \mathrm{H}), 3.83\left(\mathrm{~s}, 3 \mathrm{H}, \mathrm{OCH}_{3}\right), 4.72$ (dd, $J=9.2,7.1 \mathrm{~Hz}, 1 \mathrm{H}), 7.01-7.10(\mathrm{~m}, 2 \mathrm{H}, \mathrm{Ar}-\mathrm{H}), 7.25-7.48(\mathrm{~m}, 8 \mathrm{H}$, $\mathrm{Ar}-\mathrm{H}) \mathrm{ppm} ;{ }^{13} \mathrm{C}$ NMR $\left(75 \mathrm{MHz}, \mathrm{CDCl}_{3}\right): \delta 22.2\left(\mathrm{CH}_{3}\right), 24.4\left(\mathrm{CH}_{3}\right)$, $24.7(\mathrm{CH}), 43.2\left(\mathrm{CH}_{2}\right), 50.3(\mathrm{CH}), 52.5\left(\mathrm{CH}_{3}\right), 56.4(\mathrm{CH}), 62.3(\mathrm{CH})$, 70.5 (C), 126.1, 127.2, 128.5, 128.6, 128.7, 129.1, 131.6, 137.1 (Ar-C), 172.8 (C=0), 173.8 (C=0), 174.8 (C=0) ppm; MS (EI): m/z $350\left(\mathrm{M}^{+}-\mathrm{C}_{4} \mathrm{H}_{9}, 21 \%\right), 349$ (50), 347 (100), 233 (16), 202 (10), 190 (50), 170 (11), 147 (11), 143 (13), 130 (14), 115 (10), 103 (15). HRMS (DIP): $\mathrm{m} / \mathrm{z}\left[\mathrm{M}^{+}\right]$calculated for $\mathrm{C}_{24} \mathrm{H}_{26} \mathrm{~N}_{2} \mathrm{O}_{4}, 406.1893$; found: 406.1905 .

\section{Methyl}

6a-isobutyl-4,6-dioxo-2,5diphenyloctahydropyrrolo[3,4-b]pyrrole-3-carboxylate

(5b): After 36 hours and work up the product was isolated as a sticky yellow oil (211 mg, $52 \%$ yield); column chromatography (silica gel deactivate with $5 \% \mathrm{Et}_{3} \mathrm{~N}$ ) ( $n$-hexane:EtOAc; 8:2); IR (ATR) Umax: 29254, 2922, 1709, 1495, 1378, 1235, 1191, 734, 702, 690, 617, $586 \mathrm{~cm}^{-1}$; ${ }^{1} \mathrm{H}$ NMR (300 MHz, $\left.\mathrm{CDCl}_{3}\right): \delta 0.98$ (d, $J=6.5$ $\mathrm{Hz}, 3 \mathrm{H}$ ), 1.07 (d, $J=6.5 \mathrm{~Hz}, 3 \mathrm{H}), 1.79-1.98(\mathrm{~m}, 2 \mathrm{H}), 2.10-2.19(\mathrm{~m}$, $1 \mathrm{H}), 3.64(\mathrm{dd}, J=4.7,3.3 \mathrm{~Hz}, 1 \mathrm{H}), 3.76(\mathrm{~d}, J=3.3 \mathrm{~Hz}, 1 \mathrm{H}), 3.79$ (s, $\left.3 \mathrm{H}, \mathrm{OCH}_{3}\right), 4.79$ (d, J = $\left.4.7 \mathrm{~Hz}, 1 \mathrm{H}\right), 6.80-6.90(\mathrm{~m}, 2 \mathrm{H}, \mathrm{Ar}-\mathrm{H}), 7.23-$ $7.67(\mathrm{~m}, 8 \mathrm{H}, \mathrm{Ar}-\mathrm{H}) \mathrm{ppm} ;{ }^{13} \mathrm{C}$ NMR $\left(75 \mathrm{MHz}, \mathrm{CDCl}_{3}\right): \delta 23.4\left(\mathrm{CH}_{3}\right)$, $24.3\left(\mathrm{CH}_{3}\right), 25.6(\mathrm{CH}), 43.2\left(\mathrm{CH}_{2}\right), 51.2(\mathrm{CH}), 52.9\left(\mathrm{CH}_{3}\right), 54.7(\mathrm{CH})$, $65.8(\mathrm{CH}), 69.9$ (C), 126.4, 128.1, 128.8, 128.9, 129.1, 131.8 (ArC), $173.1(\mathrm{C}=0), 175.9$ (C=0), 178.2 (C=0) ppm; MS (EI): m/z 350 ( $\left.\mathrm{M}^{+}-\mathrm{C}_{4} \mathrm{H}_{9}, 36 \%\right), 318$ (13), 200 (94), 191 (20), 177 (100), 171 (13), 144 (21), 143 (14), 119 (14), 91 (21). HRMS (DIP): m/z [M+] calculated for $\mathrm{C}_{24} \mathrm{H}_{26} \mathrm{~N}_{2} \mathrm{O}_{4}$, 406.1893; found: 406.1868 .

Methyl 1-isobutyl-4,6-dioxo-5-phenyl-3-(pyridin-2yl)octahydropyrrolo[3,4-c]pyrrole-1-carboxylate (4c): After 18 hours and work up the product was isolated as a white solid (350 mg, $86 \%$ yield); column chromatography ( $n$-hexane:EtOAc; 6:4); mp: 171-175 으. IR (ATR) U $\cup_{\max }: 1705.7,1387,1248,1207$,
1151, 1181, 764, 728, $691 \mathrm{~cm}^{-1} ;{ }^{1} \mathrm{H}$ NMR (300 MHz, $\left.\mathrm{CDCl}_{3}\right): \delta 0.88$ (d, $J=6.6 \mathrm{~Hz}, 3 \mathrm{H}$ ), 1.01 (d, $J=6.7 \mathrm{~Hz}, 3 \mathrm{H}$ ), 1.69 (dd, $J=14.1,4.7 \mathrm{~Hz}$, $1 \mathrm{H}), 1.81-1.97(\mathrm{~m}, 1 \mathrm{H}), 2.16(\mathrm{~m}, 1 \mathrm{H}), 3.46(\mathrm{~d}, J=7.6 \mathrm{~Hz}, 1 \mathrm{H}), 3.70$ (dd, $J=9.0,7.6 \mathrm{~Hz}, 1 \mathrm{H}), 3.86\left(\mathrm{~s}, \mathrm{OCH}_{3}\right), 4.70(\mathrm{~d}, J=9.0 \mathrm{~Hz}, 1 \mathrm{H})$, 7.02-7.25 (m, 3H, Ar-H), 7.30-7.49 (m, 4H, Ar-H), 7.68-7.73 (m, $1 \mathrm{H}, \mathrm{Ar}-\mathrm{H}), 8.34-8.65(\mathrm{~m}, 1 \mathrm{H}, \mathrm{Ar}-\mathrm{H}) \mathrm{ppm} ;{ }^{13} \mathrm{C}$ NMR $(75 \mathrm{MHz}$, $\left.\mathrm{CDCl}_{3}\right): \delta 22.1\left(\mathrm{CH}_{3}\right), 24.4\left(\mathrm{CH}_{3}\right), 25.0(\mathrm{CH}), 44.3\left(\mathrm{CH}_{2}\right), 51.7(\mathrm{CH})$, $52.6\left(\mathrm{CH}_{3}\right), 58.5(\mathrm{CH}), 65.0(\mathrm{CH}), 72.2(\mathrm{C}), 123.7,123.9,126.6$, 128.7, 129.1, 131.9, 136.9, 149.4, 155.5 (Ar-C), 172.33 (C=0), $174.5(\mathrm{C}=0) 174.8(\mathrm{C}=0)$ ppm; MS (EI): $\mathrm{m} / \mathrm{z} 408\left(\mathrm{M}^{+} 12 \%\right), 407$ (47), 351 (14), 350 (24), 349 (23), 348 (100), 177 (10), 175 (41), 171 (17), 145 (18), 131 (13). HRMS (DIP): $\mathrm{m} / \mathrm{z}$ [M+] calculated for $\mathrm{C}_{23} \mathrm{H}_{25} \mathrm{~N}_{3} \mathrm{O}_{4}$ : 407.1845; found: 407.1851 .

Methyl 6a-isobutyl-4,6-dioxo-5-phenyl-2-(pyridin-2yl)octahydropyrrolo[3,4-b]pyrrole-3-carboxylate (5c): After 37 hours and work up the product was isolated as a sticky yellow oil (157 mg, $40 \%$ yield); column chromatography (silica gel deactivate with $5 \% \mathrm{Et}_{3} \mathrm{~N}$ ) ( $n$-hexane:EtOAc; 6:4); IR (ATR) U $\max$ : 3321, 2957, 2925, 1709, 1593, 1375, 1191, 1138, 749, 690, 599 $\mathrm{cm}^{-1}$; ${ }^{1} \mathrm{H}$ NMR (300 MHz, MeOD): $\delta 0.94(\mathrm{~d}, J=6.5 \mathrm{~Hz}, 3 \mathrm{H}), 1.02(\mathrm{~d}$, $J=6.6 \mathrm{~Hz}, 3 \mathrm{H}), 1.72-1.99(\mathrm{~m}, 2 \mathrm{H}), 2.02-2.23(\mathrm{~m}, 1 \mathrm{H}), 3.79-3.83(\mathrm{~m}$, 1H), $3.93(\mathrm{~d}, J=2.7 \mathrm{~Hz}, 1 \mathrm{H}), 4.93(\mathrm{~d}, J=3.3 \mathrm{~Hz}, 1 \mathrm{H}), 6.73-6.94(\mathrm{~m}$, 2H, Ar-H), 7.20-7.43 (m, 4H, Ar-H), 7.60 (d, J= 7.9 Hz, 1H, Ar-H), 7.69-7.74 (m, 1H, Ar-H), 8.40-8.46 (m, 1H, Ar-H) ppm; ${ }^{13} \mathrm{C} \mathrm{NMR}$ (75 MHz, MeOD): $\delta 24.0\left(\mathrm{CH}_{3}\right), 24.7\left(\mathrm{CH}_{3}\right), 26.5(\mathrm{CH}), 45.4\left(\mathrm{CH}_{2}\right)$, $53.5(\mathrm{CH}), 57.4(\mathrm{CH}), 68.3(\mathrm{CH}), 71.8(\mathrm{C}), 122.9,123.8,127.5$, 129.6, 129.9, 133.3, 138.7, 149.8, 162.5 (Ar-C), 178.6 (C=0), 178.6

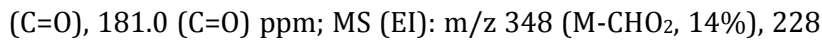
(100), 227 (36), 171 (24), 145 (36), 119 (56), 92 (43), 91 (25), 77 (22), 44 (14). HRMS (DIP): $\mathrm{m} / \mathrm{z}$ [M-CHO ${ }_{2}$ calculated for $\mathrm{C}_{21} \mathrm{H}_{22} \mathrm{~N}_{3} \mathrm{O}_{2}$ : 348.1692; found: 348.1712 .

Methyl 1-isobutyl-4,6-dioxo-5-phenyl-3-(thiophen-2yl)octahydropyrrolo[3,4-c]pyrrole-1-carboxylate (4d): After 19 hours and work up the product was isolated as a red pale solid (301 mg, $73 \%$ yield); column chromatography ( $n$-hexane:EtOAc; 6:4); mp: 139 - $143^{\circ} \mathrm{O}$. IR (ATR) U $u_{\max }$ 1710, 1501, 1384, 1236, $1208,1177,1164,822,701,692 \mathrm{~cm}^{-1}$; ${ }^{1} \mathrm{H}$ NMR (300 MHz, $\left.\mathrm{CDCl}_{3}\right)$ : $\delta 0.88(\mathrm{~d}, J=6.4 \mathrm{~Hz}, 3 \mathrm{H}), 1.01(\mathrm{~d}, J=6.4 \mathrm{~Hz}, 3 \mathrm{H}), 1.61-1.75(\mathrm{~m}, 2 \mathrm{H})$, 2.03-2.24 (m, 1H), 3.37 (d, $J=7.6 \mathrm{~Hz}, 1 \mathrm{H}), 3.59$ (dd, $J=9.2,7.6 \mathrm{~Hz}$, 1H), $3.85\left(\mathrm{~s}, \mathrm{OCH}_{3}\right), 5.00(\mathrm{~d}, J=9.1 \mathrm{~Hz}, 1 \mathrm{H}), 7.01(\mathrm{dd}, J=5.1,3.6$ $\mathrm{Hz}, 1 \mathrm{Ar}-\mathrm{H}), 7.11-7.43$ (m, 7H, Ar-H) ppm; ${ }^{13} \mathrm{C}$ NMR $(101 \mathrm{MHz}$ $\left.\mathrm{CDCl}_{3}\right): \delta 22.1\left(\mathrm{CH}_{3}\right), 24.3\left(\mathrm{CH}_{3}\right), 24.4(\mathrm{CH}), 43.0\left(\mathrm{CH}_{2}\right), 50.1(\mathrm{CH})$, $52.4\left(\mathrm{CH}_{3}\right), 55.5(\mathrm{CH}), 57.9(\mathrm{CH}), 70.0(\mathrm{C}), 125.1,125.4,126.2$, 127.1, 128.5, 129.0, 131.6, 141.1 (Ar-C), 172.3 (C=0), 173.3 $(\mathrm{C}=0), 174.6$ (C=0) ppm; MS (EI): m/z 369 (M- $\left.\mathrm{C}_{3} \mathrm{H}_{7}, 2 \%\right), 357$ (5), 356 (22), 355 (34), 354 (23), 353 (100), 296 (11), 239 (45), 206 (10), 197 (9), 196 (80), 179 (26), 162 (11), 149 (12) 136 (17), 109 (15). HRMS (DIP): $\mathrm{m} / \mathrm{z}\left[\mathrm{M}^{+}\right]$calculated for $\mathrm{C}_{22} \mathrm{H}_{24} \mathrm{~N}_{2} \mathrm{O}_{4} \mathrm{~S}$ : 412.1457; found: 412.1469.

Methyl 6a-isobutyl-4,6-dioxo-5-phenyl-2-(thiophen-2yl)octahydropyrrolo[3,4-b]pyrrole-3-carboxylate (5d): After 36 hours and work up the product was isolated as a sticky yellow oil (175 mg, $44 \%$ yield); column chromatography (silica gel deactivate with $5 \% \mathrm{Et}_{3} \mathrm{~N}$ ) ( $n$-hexane:EtOAc; 6:4); IR (ATR) U max: 3340, 2956, 2926, 1708, 1378, 1198, 1139, 843, 689, $597 \mathrm{~cm}^{-1}$; ${ }^{1} \mathrm{H}$ NMR (300 MHz, MeOD): $\delta 0.95(\mathrm{~d}, J=6.4 \mathrm{~Hz}, 3 \mathrm{H}), 1.04(\mathrm{~d}, J=6.4$ $\mathrm{Hz}, 3 \mathrm{H}), 1.71-2.00(\mathrm{~m}, 2 \mathrm{H}), 2.03-2.16(\mathrm{~m}, 1 \mathrm{H}), 3.66$ (dd, $J=4.7,2.2$ $\mathrm{Hz}, 1 \mathrm{H}), 3.89$ (d, $J=2.3 \mathrm{~Hz}, 1 \mathrm{H}), 5.05(\mathrm{~m}, 1 \mathrm{H}), 6.76-6.69(\mathrm{~m}, 2 \mathrm{H}$, $\mathrm{Ar}-\mathrm{H}), 6.92(\mathrm{dd}, J=5.1,3.5 \mathrm{~Hz}, 1 \mathrm{Ar}-\mathrm{H}), 6.98$ (d, $J=3.5 \mathrm{~Hz}, 1 \mathrm{Ar}-\mathrm{H})$, 
7.25-7.43 (m, 4H, Ar-H) ppm; ${ }^{13} \mathrm{C}$ NMR (75 MHz MeOD): $\delta 23.9$ $\left(\mathrm{CH}_{3}\right), 24.7\left(\mathrm{CH}_{3}\right), 26.5(\mathrm{CH}), 44.7\left(\mathrm{CH}_{2}\right), 52.5(\mathrm{CH}), 53.5(\mathrm{CH}), 63.7$ (CH), 71.9 (C), 121.0, 125.0, 126.2, 127.8, 128.2, 129.8, 133.3, 150.5 (Ar-C), 178.6 (2x C=0), 181.0 (C=0) ppm; MS (EI): m/z 310 (M- $\left.\mathrm{C}_{4} \mathrm{H}_{8} \mathrm{O}_{2}\right), 278$ (11), 277 (11), 251 (14), 209 (15), 207 (15), 206 (100), 183 (23), 169 (80), 150 (19), 149 (17). HRMS (DIP): m/z $\left[\mathrm{M}^{+}\right]$calculated for $\mathrm{C}_{22} \mathrm{H}_{24} \mathrm{~N}_{2} \mathrm{O}_{4} \mathrm{~S}: 412.1457$; found: 412.1452 .

\section{Methyl \\ 1-benzyl-4,6-dioxo-3,5-} diphenyloctahydropyrrolo[3,4-c]pyrrole-1-carboxylate

(4e): After 18 hours and work up the product was isolated as a white solid (356 mg, $81 \%$ yield); column chromatography ( $n$ hexane:EtOAc; 8:2); mp: 231-234 ํㅡ. IR (ATR) Umax: 1750, 1716, $1493,1380,1209,1178,1101,853,724,703,661 \mathrm{~cm}^{-1}$; ${ }^{1} \mathrm{H}$ NMR (300 MHz, $\left.\mathrm{CDCl}_{3}\right): \delta 2.35($ br s,1H, NH), $3.11(\mathrm{~d}, J=13.5 \mathrm{~Hz}, 1 \mathrm{H})$ 3.49 (d, $J=13.3 \mathrm{~Hz}, 1 \mathrm{H}$ ), 3.61 (d, $J=7.6 \mathrm{~Hz}, 1 \mathrm{H}), 3.70$ (dd, $J=9.4$, $7.6 \mathrm{~Hz}, 1 \mathrm{H}), 3.86\left(\mathrm{~s}, 3 \mathrm{H}, \mathrm{OCH}_{3}\right), 4.96(\mathrm{~d}, J=9.4 \mathrm{~Hz}, 1 \mathrm{H}), 6.94-7.05$ (m, 2H, Ar-H), 7.11-7.18 (m, 2H, Ar-H), 7.25-7.41 (m, 9H, Ar-H), 7.48-7.57 (m, 2H, Ar-H) ppm; ${ }^{13} \mathrm{C}$ NMR $\left(101 \mathrm{MHz}, \mathrm{CDCl}_{3}\right): \delta 40.4$ $\left(\mathrm{CH}_{2}\right), 49.15(\mathrm{CH}), 52.4\left(\mathrm{CH}_{3}\right), 54.3(\mathrm{CH}), 61.3(\mathrm{CH}), 71.3(\mathrm{C})$ $126.1,127.4,127.6,128.5,128.6,128.6,128.9,129.0,129.5$, 131.4, 134.8, 137.2 (Ar-C), 171.5 (C=0), 173.8 (C=0), 174.9 (C=0) ppm; MS (EI): m/z 381 (M- $\left.\mathrm{C}_{2} \mathrm{H}_{3} \mathrm{O}_{2}, 3 \%\right), 350$ (22), 349 (100), 202 (14), 170 (13), 143 (11), 91 (15). HRMS (DIP): m/z [M+] calculated for $\mathrm{C}_{27} \mathrm{H}_{24} \mathrm{~N}_{2} \mathrm{O}_{4}$, 440.1736; found: 440.1755 .

\section{Methyl 6a-benzyl-4,6-dioxo-2,5-} diphenyloctahydropyrrolo[3,4-b]pyrrole-3-carboxylate

(5e): After 37 hours and work up the product was isolated as a sticky yellow oil (194 mg, $44 \%$ yield); column chromatography (silica gel deactivate with $5 \% \mathrm{Et}_{3} \mathrm{~N}$ ) ( $n$-hexane:EtOAc; 8:2); IR (ATR) U $\max$ : 2918, 2849, 1711, 1455, 1377, 1259, 1173, 1028, 732, 700, 691, $587 \mathrm{~cm}^{-1} ;{ }^{1} \mathrm{H}$ NMR (300 MHz, $\left.\mathrm{CDCl}_{3}\right): \delta 3.16(\mathrm{~d}, J=12.8$ $\mathrm{Hz}, 1 \mathrm{H}), 3.63$ (d, $J=12.8 \mathrm{~Hz}, 1 \mathrm{H}), 3.67$ (dd, $J=4.0,2.9 \mathrm{~Hz}, 1 \mathrm{H}), 3.77$ $(\mathrm{d}, J=2.9 \mathrm{~Hz}, 1 \mathrm{H}), 3.83\left(\mathrm{~s}, 3 \mathrm{H}, \mathrm{OCH}_{3}\right), 4.88(\mathrm{~d}, J=4.0 \mathrm{~Hz}, 1 \mathrm{H}), 6.37-$ $6.60(\mathrm{~m}, 2 \mathrm{H}, \mathrm{Ar}-\mathrm{H}), 7.27-7.36(\mathrm{~m}, 11 \mathrm{H}, \mathrm{Ar}-\mathrm{H}), 7.39-7.45(\mathrm{~m}, 2 \mathrm{H}$, $\mathrm{Ar}-\mathrm{H}) \mathrm{ppm} ;{ }^{13} \mathrm{C}$ NMR $\left(126 \mathrm{MHz}, \mathrm{CDCl}_{3}\right): \delta 40.7\left(\mathrm{CH}_{2}\right), 50.3(\mathrm{CH})$, $53.0\left(\mathrm{CH}_{3}\right), 54.2(\mathrm{CH}), 66.4(\mathrm{CH}), 71.6(\mathrm{C}), 126.4,126.5,127.8$, 128.2, 128.8, 129.0, 129.1, 130.5, 131.6, 134.9 (Ar-C), 173.1 $(\mathrm{C}=0), 175.3$ (C=0), 178.0 (C=0) ppm; MS (EI): m/z $349\left(\mathrm{M}-\mathrm{C}_{7} \mathrm{H}_{7}\right.$, 14\%), 317 (39), 289 (35), 234 (21), 178 (12), 177 (100), 170 (19), 143 (12), 115 (16), 91 (43). HRMS (DIP): m/z [M+] calculated for $\mathrm{C}_{27} \mathrm{H}_{24} \mathrm{~N}_{2} \mathrm{O}_{4}$, 440.1736; found: 440.1697 .

\section{Methyl 1-benzyl-4,6-dioxo-5-phenyl-3-(pyridin-2-} yl)octahydropyrrolo[3,4-c]pyrrole-1-carboxylate (4f): After 18 hours and work up the product was isolated as a white solid (388 mg, $87 \%$ yield); column chromatography ( $n$-hexane:EtOAc; 6:4); mp: 197-200 으. IR (ATR) $u_{\max }: 1710,1495,1395,1212$, 1137, 1104, 1090, 859, 767, $\left.729 \mathrm{~cm}^{-1} ;{ }^{1} \mathrm{H} \mathrm{NMR} \mathrm{(300} \mathrm{MHz,} \mathrm{CDCl}_{3}\right)$ : $3.11(\mathrm{~d}, J=13.7 \mathrm{~Hz}, 1 \mathrm{H}), 3.42(\mathrm{~d}, J=13.7 \mathrm{~Hz}, 1 \mathrm{H}), 3.63-.77(\mathrm{~m}, 2 \mathrm{H})$, $3.85\left(\mathrm{~s}, 3 \mathrm{H}, \mathrm{OCH}_{3}\right), 4.83$ (d, J = 8.9 Hz, 1H), 7.04-7.16 (m, 2H, ArH), 7.20-7.47 (m, 10H, Ar-H), 7.66 (td, $J=7.7,1.8 \mathrm{~Hz}, 1 \mathrm{H}, \mathrm{Ar}-\mathrm{H})$, 8.53 (ddd, $J=4.9,1.8,0.9 \mathrm{~Hz}, 1 \mathrm{H}, \mathrm{Ar}-\mathrm{H}) \mathrm{ppm} ;{ }^{13} \mathrm{C}$ NMR $(101 \mathrm{MHz}$, $\left.\mathrm{CDCl}_{3}\right)$ : $\delta 42.0\left(\mathrm{CH}_{2}\right), 51.4(\mathrm{CH}), 52.7\left(\mathrm{CH}_{3}\right), 57.1(\mathrm{CH}), 64.8(\mathrm{CH})$, 73.5 (C), 123.6, 123.7, 126.5, 127.3, 128.5, 128.7, 129.1, 130.2, 131.8, 135.8, 136.9, 149.3, 156.0 (Ar-C), $171.1(\mathrm{C}=0), 174.3$ (C=0), 174.9 (C=0) ppm; MS (EI): m/z $382\left(\mathrm{M}-\mathrm{C}_{2} \mathrm{H}_{3} \mathrm{O}_{2}, 4 \%\right), 351$ (21), 350 (100), 193 (4). 177 (17), 171 (23), 145 (23), 143 (4), 117 (6), 116 (5), 91 (13). HRMS (DIP): m/z [M+] calculated for $\mathrm{C}_{26} \mathrm{H}_{23} \mathrm{~N}_{3} \mathrm{O}_{4}, 441.1689$; found 441.1669 .
Methyl 6a-benzyl-4,6-dioxo-5-phenyl-2-(pyridin-2yl)octahydropyrrolo[3,4-b]pyrrole-3-carboxylate (5f): After 36 hours and work up the product was isolated as a Sticky yellow oil (238 mg, $54 \%$ yield); column chromatography (silica gel deactivate with $5 \% \mathrm{Et}_{3} \mathrm{~N}$ ) ( $n$-hexane:EtOAc; 6:4); IR (ATR) U $\max$ : 2923, 2853, 1709, 1592, 1378, 1178, 1051, 744, 702, $590 \mathrm{~cm}^{-1}$; ${ }^{1} \mathrm{H}$ NMR (300 MHz, $\left.\mathrm{CDCl}_{3}\right): 3.18(\mathrm{~d}, J=12.8 \mathrm{~Hz}, 1 \mathrm{H}), 3.60(\mathrm{~d}, J=$ $12.9 \mathrm{~Hz}, 1 \mathrm{H}), 3.87\left(\mathrm{~s}, 3 \mathrm{H}, \mathrm{OCH}_{3}\right), 3.89(\mathrm{~d}, J=2.0 \mathrm{~Hz}, 1 \mathrm{H}), 4.11-4.15$ (m, 1H), $5.04(\mathrm{~d}, J=2.7 \mathrm{~Hz}, 1 \mathrm{H}), 6.48-6.70(\mathrm{~m}, 2 \mathrm{H}, \mathrm{Ar}-\mathrm{H}), 6.90-7.53$ (m, 9H, Ar-H), 7.54 (d, J= 7.8 Hz, 1H, Ar-H), 7.66-7.77 (m, 1H, ArH), 8.49 (ddd, $J=4.9 \mathrm{~Hz}, 1 \mathrm{H}, \mathrm{Ar}-\mathrm{H}$ ) ppm; ${ }^{13} \mathrm{C}$ NMR $(126 \mathrm{MHz}$, $\left.\mathrm{CDCl}_{3}\right): \delta 41.8\left(\mathrm{CH}_{2}\right), 49.9(\mathrm{CH}), 52.5(\mathrm{CH}), 53.19\left(\mathrm{CH}_{3}\right), 67.1(\mathrm{CH})$, 72.3 (C), 121.6, 123.1, 126.1, 127.7, 128.6, 128.9, 130.4, 131.5, 135.0, 138.0, 148.7, 159.2 (Ar-C), 173.1 (C=0), 175.2 (C=0), 178.3 (C=0) ppm;MS (EI): m/z $382\left(\mathrm{M}^{+}-\mathrm{C}_{2} \mathrm{H}_{3} \mathrm{O}_{2}, 51 \%\right), 350$ (100), 235 (14), 177 (19), 171 (22), 145 (24), 119 (28), 117 (19), 93 (21), 92(22), 91 (52), 78 (14), 44 (23). HRMS (DIP): m/z [M+] calculated for $\mathrm{C}_{26} \mathrm{H}_{23} \mathrm{~N}_{3} \mathrm{O}_{4}, 441.1689$; found 441.1698.

Methyl (1S,3R)-1-((1H-indol-3-yl)methyl)-5-methyl-4,6dioxo-3-phenyloctahydropyrrolo [3,4-c]pyrrole-1carboxylate (4gb): After $26 \mathrm{~h}$ and work up the product $(317 \mathrm{mg}$, $76 \%$ yield) was crystallised as colourless prisms: $\mathrm{mp} 232-234{ }^{\circ} \mathrm{C}$ (dec.); IR $v_{\text {max: }}$ 3358, 2981, 2884, 1776, 1732, 1685, 1440, 1387, 1285, 1200, 1103, 1078, 963, 843, 727, 701, $654 \mathrm{~cm}^{-1}$; ${ }^{1} \mathrm{H}$ NMR (400 MHz, DMSO): $\delta 10.98$ (bs, 1H, NH), $7.55(\mathrm{~d}, 1 \mathrm{H}, J=7.88 \mathrm{~Hz}$, Ar-H), 7.36-6.96 (m, 9H, Ar-H), 5.00 (dd, $1 \mathrm{H}, J=9.40 \mathrm{~Hz}, 5.16 \mathrm{~Hz}$, 5-H), 3.74 (dd, $1 \mathrm{H}, J=9.20 \mathrm{~Hz}, 7.64 \mathrm{~Hz}, 4-\mathrm{H}), 3.69$ (s, $3 \mathrm{H}, \mathrm{OCH}_{3}$ ), 3.62 (d, $1 \mathrm{H}, J=7.40 \mathrm{~Hz}, 3-\mathrm{H}), 3.44(\mathrm{~d}, 1 \mathrm{H}, J=14.56 \mathrm{~Hz}, 6-\mathrm{H}), 3.34$ (d, $\left.1 \mathrm{H}, J=14.50 \mathrm{~Hz}, 6-\mathrm{H}^{\prime}\right), 2.66\left(\mathrm{~s}, 3 \mathrm{H}, \mathrm{NCH}_{3}\right), 2.36$ (d, $1 \mathrm{H}, J=5.02$ $\mathrm{Hz}, \mathrm{NH}) \mathrm{ppm} ;{ }^{13} \mathrm{C}$ NMR (100 MHz, DMSO): $\delta 176.1$ (C=0), 174.9 $(\mathrm{C}=0), 171.7(\mathrm{C}=0), 139.1,135.9,127.9$ ( $2 \times \mathrm{C}), 127.5,127.4$, 127.3 ( 2 x C), 124.3, 120.9, 118.5, 118.1, 111.4, 107.9, 70.2, 59.7, 53.78, 51.5, 49.0, 30.2, 24.2 ppm; MS (ESI, M+H+): m/z 418.3 $\left(\mathrm{M}+\mathrm{H}^{+}, 100\right)$; HRMS (DIP): $\mathrm{m} / \mathrm{z}\left[\mathrm{M}^{+}\right]$calculated for $\mathrm{C}_{24} \mathrm{H}_{23} \mathrm{~N}_{3} \mathrm{O}_{4}$, 417.1694; found: 417.1689.

Methyl (1S,3R)-1-((1H-indol-3-yl)methyl)-4,6-dioxo-5phenyl-3-(pyridin-2-yl) octahydropyrrolo [3,4-c] pyrrole-1carboxylate (4gc): After $26 \mathrm{~h}$ and work up the product (384 mg, $80 \%$ yield) was isolated as colourless prisms: mp 231-233 ${ }^{\circ} \mathrm{C}$ (dec.); IR $v_{\text {max: }}$ 3381, 3350, 3061, 2959, 2878, 1779, 1707, 1614, 1591, 1489, 1435, 1384, 1323, 1204, 1178, 1101, 739, $686 \mathrm{~cm}^{-1}$; ${ }^{1} \mathrm{H}$ NMR (400 MHz, DMSO): $\delta 10.86$ (bs, $\left.1 \mathrm{H}, \mathrm{NH}\right), 8.59-8.57$ (m, $1 \mathrm{H}, \mathrm{Ar}-\mathrm{H}), 7.85-6.97(\mathrm{~m}, 13 \mathrm{H}, \mathrm{Ar}-\mathrm{H}), 5.16(\mathrm{dd}, 1 \mathrm{H}, J=11.22 \mathrm{~Hz}$, $9.26 \mathrm{~Hz}, 5-\mathrm{H}$ ), 3.97 (dd, $1 \mathrm{H}, J=9.16 \mathrm{~Hz}, 7.64 \mathrm{~Hz}, 4-\mathrm{H}), 3.89$ (d, $1 \mathrm{H}$, $J=7.60 \mathrm{~Hz}, 3-\mathrm{H}), 3.79(\mathrm{~d}, 1 \mathrm{H}, J=11.24 \mathrm{~Hz}, \mathrm{NH}), 3.68\left(\mathrm{~s}, 3 \mathrm{H}, \mathrm{OCH}_{3}\right)$, 3.47 (d, $1 \mathrm{H}, J=14.68 \mathrm{~Hz}, 6-\mathrm{H}), 3.30$ (d, $\left.1 \mathrm{H}, J=14.60 \mathrm{~Hz}, 6-\mathrm{H}^{\prime}\right)$ ppm; ${ }^{13} \mathrm{C}$ NMR (100 MHz, DMSO): $\delta 175.2(\mathrm{C}=0), 174.4(\mathrm{C}=0), 171.7$ $(\mathrm{C}=0), 156.4,148.8,136.8,135.6,132.2,128.8$ (2 x C), 128.2, 128.1, 126.7 ( 2 x C), 124.3, 123.9, 123.3, 120.6, 118.6, 118.2, 111.2, 109.2, 70.0, 63.4, 57.4, 51.8, 51.4, 31.1 ppm; MS (ESI, $\left.\mathrm{M}+\mathrm{H}^{+}\right): \mathrm{m} / \mathrm{z} 481.2\left(\mathrm{M}+\mathrm{H}^{+}, 100\right)$; HRMS (DIP): $\mathrm{m} / \mathrm{z}\left[\mathrm{M}^{+}\right.$] calculated for $\mathrm{C}_{28} \mathrm{H}_{24} \mathrm{~N}_{4} \mathrm{O}_{4}$, 480.1798; found: 480.1702

Methyl(1S,3R)-1-((1H-indol-3-yl)methyl)-5-methyl-4,6dioxo-3-(pyridin-2-yl) octahydropyrrolo [3,4-c]pyrrole-1carboxylate (4gd): After $26 \mathrm{~h}$ and work up the product (313 mg, $75 \%$ yield) was isolated and crystallised as colourless prisms: mp 229-231 ㅇ (dec.); IR $v_{\max }$ 3359, 3300, 2981, 1774, 1735, $1682,1595,1443,1289,1224,1095,995,727 \mathrm{~cm}^{-1} ;{ }^{1} \mathrm{H}$ NMR $(400$ MHz, DMSO): 10.82 (bs, 1H, NH), 8.49-8.44 (m, 1H, Ar-H), 7.785- 
6.92 (m, 8H, Ar-H), 5.02 (dd, 1H, J=11.04 Hz, $9.32 \mathrm{~Hz}, 5-\mathrm{H}$ ), 3.97 (dd, $1 \mathrm{H}, J=9.14 \mathrm{~Hz}, 7.54 \mathrm{~Hz}, 4-\mathrm{H}$ ), 3.67 (s, 3H, OCH $\left.H_{3}\right), 3.65$ (d, $1 \mathrm{H}$, $J=7.48 \mathrm{~Hz}, 3-\mathrm{H}), 3.55$ (d, 1H, $J=11.12 \mathrm{~Hz}, \mathrm{NH}), 3.39$ (d, $1 \mathrm{H}, J=$ $14.92 \mathrm{~Hz}, 6-\mathrm{H}), 3.21\left(\mathrm{~d}, 1 \mathrm{H}, J=14.84 \mathrm{~Hz}, 6-\mathrm{H}^{\prime}\right), 2.61\left(\mathrm{~s}, 3 \mathrm{H}, \mathrm{NCH}_{3}\right)$ ppm; ${ }^{13} \mathrm{C}$ NMR (100 MHz, DMSO): $\delta 176.0(\mathrm{C}=0), 175.2(\mathrm{C}=0)$, 171.7 (C=0), 156.4, 148.7, 136.6, 135.5, 128.0, 124.2, 123.7, 123.0, 120.6, 188.5, 118.2, 111.1, 109.1, 72.6, 62.9, 57.0, 51.8, 51.3, 30.9, 24.3 ppm; MS (ESI, $\left.\mathrm{M}+\mathrm{H}^{+}\right): \mathrm{m} / \mathrm{z} 419.2\left(\mathrm{M}+\mathrm{H}^{+}, 100\right)$; HRMS (DIP): $\mathrm{m} / \mathrm{z}\left[\mathrm{M}^{+}\right]$calculated for $\mathrm{C}_{23} \mathrm{H}_{22} \mathrm{~N}_{4} \mathrm{O}_{4}, 418.1641$; found: 418.1642 .

\section{Methyl(1S,3R)-1-((1H-indol-3-yl)methyl)-3-(3-} chlorophenyl)-4,6-dioxo-5-phenyloctahydropyrrolo [3,4-c] pyrrole-1-carboxylate (4ge): After $26 \mathrm{~h}$ and work up the product (349 mg, $68 \%$ yield) was isolated and crystallised as colourless prisms: mp 273-275 으 (dec.); IR $v_{\max }$ : 3335, 2981, 1779, 1708, 1598, 1573, 1433, 1385, 1202, 1181, 1100, 954, 748, $689 \mathrm{~cm}^{-1}$; ${ }^{1} \mathrm{H}$ NMR (400 MHz, DMSO): $\delta 11.02$ (bs, 1H, NH), 7.58$6.98(\mathrm{~m}, 9 \mathrm{H}, \mathrm{Ar}-\mathrm{H}), 5.14$ (dd, 1H, $J=9.46 \mathrm{~Hz}, 4.50 \mathrm{~Hz}, 5-\mathrm{H}), 3.91$ (dd, $1 \mathrm{H}, J=9.48 \mathrm{~Hz}, 7.64 \mathrm{~Hz}, 4-\mathrm{H}$ ), 383 (dd, $1 \mathrm{H}, J=7.56 \mathrm{~Hz}, 1.60$ $\mathrm{Hz}, 3-\mathrm{H}$ ), 3.66 (s, 3H, OCH3), 3.50 (d, 1H, J = $14.56 \mathrm{~Hz}, 6-\mathrm{H}), 3.35$ (d, $\left.1 \mathrm{H}, J=14.52 \mathrm{~Hz}, 6-\mathrm{H}^{\prime}\right), 2.69$ (d, $1 \mathrm{H}, J=4.36 \mathrm{~Hz}, \mathrm{NH}$ ) ppm; ${ }^{13} \mathrm{C}$ NMR (100 MHz, DMSO): $\delta 175.3(\mathrm{C}=0), 174.0(\mathrm{C}=0), 171.6(\mathrm{C}=0)$, $142.0,135.9,132.7,132.0,129.8,128.8$ ( 2 x C) $128.2,127.5(2 \mathrm{x}$ C), 127.1, 126.5 ( 2 x C), 126.4, 124.6, 121.0, 118.5, 118.0, 111.4, 107.6, 70.3, 59.0, 53.7, 51.5, 48.7, 30.3 ppm; MS (ESI, M+H+): m/z $512.2\left(\mathrm{M}+\mathrm{H}^{+}, 100\right), 514.2\left(\mathrm{M}+\mathrm{H}^{+}, 35\right)$; HRMS (DIP): $\mathrm{m} / \mathrm{z}\left[\mathrm{M}^{+}\right]$ calculated for $\mathrm{C}_{29} \mathrm{H}_{24} \mathrm{ClN}_{3} \mathrm{O}_{4}, 513.1455$; found: 513.1442

\section{Methyl(1S,3R)-1-((1H-indol-3-yl)methyl)-3-(3-} chlorophenyl)-5-methyl-4,6-dioxooctahydropyrrolo[3,4c]pyrrole-1-carboxylate (4gf): After $26 \mathrm{~h}$ and work up the product ( $307 \mathrm{mg}, 68 \%$ yield) was isolated and crystallised as colourless prisms: $m p$ 251-253 으 (dec.); IR $v_{\max }$ : 3339, 3324, 3062, 2949, 2926, 1782, 1704, 1672, 1426, 1290, 1203, 1099, 1081,755, $748 \mathrm{~cm}^{-1}$; ${ }^{1} \mathrm{H}$ NMR (400 MHz, $\delta 10.99$ (bs, 1H, NH), 7.54-6.96 (m, 9H, Ar-H), 5.01 (dd, 1H, $J=9.38 \mathrm{~Hz}, 4.66 \mathrm{~Hz}, 5-\mathrm{H})$, 3.75 (dd, $1 \mathrm{H}, J=9.30 \mathrm{~Hz}, 7.54 \mathrm{~Hz}, 4-\mathrm{H}), 3.68$ (s, $\left.3 \mathrm{H}, \mathrm{OCH}_{3}\right), 3.62$ (dd, $1 \mathrm{H}, J=7.40 \mathrm{~Hz}, 1.32 \mathrm{~Hz}, 3-\mathrm{H}$ ), 3.45 (d, 1H, J=14.60 Hz, 6-H), 3.29 (d, $\left.1 \mathrm{H}, J=14.56 \mathrm{~Hz}, 6-\mathrm{H}^{\prime}\right) 2.66\left(\mathrm{~s}, 3 \mathrm{H}, \mathrm{NCH}_{3}\right) \mathrm{ppm} ;{ }^{13} \mathrm{C} \mathrm{NMR}$ (100 MHz, DMSO): $\delta 176.0(\mathrm{C}=0), 174.9(\mathrm{C}=0), 171.5(\mathrm{C}=0)$, $142.0,135.9,132.5,129.7,127.4,127.3,127.9,126.0,124.5$, 121.0, 118.5, 118.0, 111.4, 107.7, 70.1, 58.8, 53.4, 51.5, 48.7, 30.2, 24.2 ppm; MS (ESI, M+H'): m/z $452.2\left(\mathrm{M}+\mathrm{H}^{+}, 100\right), 454.2\left(\mathrm{M}+\mathrm{H}^{+}\right.$, 35); HRMS (DIP): $\mathrm{m} / \mathrm{z}\left[\mathrm{M}^{+}\right]$calculated for $\mathrm{C}_{24} \mathrm{H}_{22} \mathrm{ClN}_{3} \mathrm{O}_{4}$, 451.1299; found: 451.1293 .

\section{(1S,3R)-methyl1-((1H-indol-3-yl)methyl)-3-(4-} chlorophenyl)-5-ethyl-4,6-dioxo-octahydropyrrolo[3,4c]pyrrole-1-carboxylate (4gg): After $26 \mathrm{~h}$ and work up the product (340 mg, $73 \%$ yield) was isolated and crystallised as colourless prisms: $m p$ 237-239 ${ }^{\circ} \mathrm{C}$ (dec.); IR $\nu_{\max }: 3339,2981$, 2944, 2840, 1774 (C=0), 1739 (C=0), 1683 (C=0), 744 $\mathrm{cm}^{-1} ;{ }^{1} \mathrm{H}$ NMR (400 MHz, DMSO): $\delta 11.00$ (brs, 1H, NH), 7.54 (d, 1H, J= 7.88 $\mathrm{Hz}, \mathrm{Ar} H$ ), 7.35-7.33 (m, 5H, ArH), 7.17-6.96 (m, 3H, ArH), 5.02 (dd, $1 \mathrm{H}, J=4.68 \mathrm{~Hz}, J=9.4 \mathrm{~Hz}, 5 \mathrm{H}), 3.72\left(\mathrm{~d}, 1 \mathrm{H}, J=7.6 \mathrm{~Hz}, \mathrm{C}^{H} \mathrm{HCH}_{3}\right)$, $3.68\left(\mathrm{~s}, 3 \mathrm{H}, \mathrm{OC} \underline{H_{3}}\right), 3.60\left(\mathrm{~d}, 1 \mathrm{H}, J=7.48 \mathrm{~Hz}, \mathrm{C}^{H} \mathrm{HCH}_{3}\right), 3.43(\mathrm{~d}, 1 \mathrm{H}, J=$ $14.6 \mathrm{~Hz}, 6 H$ ), 3.30 (d, $1 \mathrm{H}, J=14.56 \mathrm{~Hz}, 6{ }^{\prime} H$ ), 3.25-3.13 (m, 2H, $3 H$ and $\mathrm{N} H), 3.44(\mathrm{~s}, 1 \mathrm{H}, \mathrm{N} H), 2.42(\mathrm{~d}, 1 \mathrm{H}, \mathrm{J}=3.56 \mathrm{~Hz}, 4 H), 0.91(\mathrm{t}, 3 \mathrm{H}$, $\left.J=7.16 \mathrm{~Hz}, \mathrm{CH}_{2} \mathrm{C}_{3}\right)$ ppm; ${ }^{13} \mathrm{C}$ NMR (100 MHz, DMSO): $\delta 175.7$ $(\mathrm{C}=0), 174.6(\mathrm{C}=0), 171.5(\mathrm{C}=0), 138.1,135.9,131.8,129.2(2 \mathrm{x}$ C), 127.7 ( $2 \times \mathrm{C}), 127.5,124.4,120.9,118.5,118.0,111.4,107.8$,
70.2, 58.8, 53.4, 51.5, 48.5, 32.9, 30.2, 12.7 ppm; MS (ESI, M+H+ m/z $466.3\left(\mathrm{M}^{+}, 100, \mathrm{Cl}: 35\right) / 468.3\left(\mathrm{M}^{+}, 33.3, \mathrm{Cl}: 37\right)-(3 / 1)$ and $467.3(\mathrm{M}+1,100, \mathrm{Cl}: 35)$ /469.3(M+1, 33.3, Cl: 37) -(3/1); HRMS (DIP): $\mathrm{m} / \mathrm{z}\left[\mathrm{M}^{+}\right]$calculated for $\mathrm{C}_{25} \mathrm{H}_{24} \mathrm{ClN}_{3} \mathrm{O}_{4}, 465.1451$; found: 465.1455 .

Methyl (2S, 3S, 6aR)-6a-((1H-indol-3-yl)methyl)-5-methyl4,6-dioxo-2-phenyl octahydro pyrrolo[3,4-b]pyrrole-3carboxylate (5gb): After $36 \mathrm{~h}$ and work up the product $(400 \mathrm{mg}$, $96 \%$ yield) was isolated and crystallised as colourless prisms: mp 151-153 으; IR $v_{\max }$ : 3355, 3059, 2981, 2889, 1710, 1595, 1495, 1436, 1383, 1195, 1011, $744 \mathrm{~cm}^{-1}$; ${ }^{1} \mathrm{H}$ NMR (400 MHz, DMSO): $\delta 11.00$ (bs, $1 \mathrm{H}, \mathrm{NH}), 7.70(\mathrm{~d}, 1 \mathrm{H}, J=7.76 \mathrm{~Hz}, \mathrm{Ar}-\mathrm{H}$ ), 7.40$7.02(\mathrm{~m}, 9 \mathrm{H}, \mathrm{Ar}-\mathrm{H}), 4.66$ (dd, 1H, $J=5.90 \mathrm{~Hz}, 5.90 \mathrm{~Hz}, 2-\mathrm{H}), 4.06$ (d, $1 \mathrm{H}, J=5.36 \mathrm{~Hz}, \mathrm{NH}), 3.54\left(\mathrm{~s}, 3 \mathrm{H}, \mathrm{OCH}_{3}\right), 3.50$ (d, $1 \mathrm{H}, J=4.96 \mathrm{~Hz}$, 4-H), 3.42 (d, 1H, J = $14.20 \mathrm{~Hz}, 6-\mathrm{H}), 3.22$ (d, 1H, J = 14.24 Hz, 6$\mathrm{H}^{\prime}$ ), $3.12(\mathrm{dd}, 1 \mathrm{H}, J=6.18 \mathrm{~Hz}, 5.14 \mathrm{~Hz}, 3-\mathrm{H}), 2.59$ (s, 3H, NCH ) ppm; ${ }^{13} \mathrm{C}$ NMR (100 MHz, DMSO): $\delta 178.9$ (C=0), 176.1 (C=0), 171.5 (C=0), 143.9, 135.9, 132.9, 130.0, 127.4, 127.3, 126.2, 125.2, 124.7, 121.0, 118.6, 118.2, 111.5, 107.6, 70.9, 65.3, 54.1, 52.1, 51.1, 28.8, 24.4 ppm; MS (ESI, $\left.\mathrm{M}+\mathrm{H}^{+}\right): \mathrm{m} / \mathrm{z} 417.4\left(\mathrm{M}+\mathrm{H}^{+}\right.$, 100); HRMS (DIP): $\mathrm{m} / \mathrm{z}$ [M+] calculated for $\mathrm{C}_{24} \mathrm{H}_{23} \mathrm{~N}_{3} \mathrm{O}_{4}, 417.1694$; found: 417.1688.

Methyl(2S,3S,6aR)-6a-((1H-indol-3-yl)methyl)-4,6-dioxo-5phenyl-2-(pyridin-2-yl)octahydropyrrolo[3,4-b]pyrrole-3carboxylate (5gc): After $36 \mathrm{~h}$ and work up the product (441 mg, $92 \%$ yield) was isolated and crystallised as colourless prisms: mp 219-221 으 (dec.); IR $\nu_{\max }$ 3352, 3058, 2981, 1712, 1595, 1541, 1436, 1383, 1099, $744 \mathrm{~cm}^{-1}$; ${ }^{1} \mathrm{H}$ NMR (400 MHz, DMSO): $\delta$ 10.91 (bs, $1 \mathrm{H}, \mathrm{NH}$ ), 8.72 (brd, $1 \mathrm{H}, J=4.16 \mathrm{~Hz}, \mathrm{Ar}-\mathrm{H}), 7.80-6.94$ (m, 13H, Ar-H), 4.45 (dd, 1H, J = 8.24 Hz, 8.20 Hz, 2-H), 3.67-3.60 (m, $2 \mathrm{H}, 3-\mathrm{H}, 6-\mathrm{H}), 3.43$ (s, 3H, OCH $), 3.39-3.30\left(\mathrm{~m}, 2 \mathrm{H}, 4-\mathrm{H}, 6-\mathrm{H}^{\prime}\right)$ ppm; ${ }^{13} \mathrm{C}$ NMR (100 MHz, DMSO): $\delta 178.8(\mathrm{C}=0), 175.1(\mathrm{C}=0)$, 172.7 (C=0), 160.2, 148.6, 136.9, 136.0, 131.6, 128.6 ( $2 \times \mathrm{C})$, 128.2, 127.4, 126.1 (2 x C), 124.7, 122.5, 121.3, 121.1, 118.7, 118.3, 111.6, 107.5, 72.5, 66.9, 52.3, 51.7, 51.1, 20.2 ppm; MS (ESI, $\left.\mathrm{M}+\mathrm{H}^{+}\right): \mathrm{m} / \mathrm{z} 481.2\left(\mathrm{M}+\mathrm{H}^{+}, 100\right)$; HRMS (DIP): $\mathrm{m} / \mathrm{z}\left[\mathrm{M}^{+}\right]$calculated for $\mathrm{C}_{28} \mathrm{H}_{24} \mathrm{~N}_{4} \mathrm{O}_{4}, 480.1798$; found: 480.1704 .

Methyl(2S,3S,6aR)-6a-((1H-indol-3-yl)methyl)-5-methyl4,6-dioxo-2-(pyridin-2-yl)octahydropyrrolo[3,4-b]pyrrole3-carboxylate (5gd): After $36 \mathrm{~h}$ and work up the product ( 355 mg, $85 \%$ yield) was isolated and crystallised as colourless prisms: $\mathrm{mp} 198-200{ }^{\circ} \mathrm{C}$ (dec.); IR $v_{\max }$ : 3355, 2981, 2972, 2889, 1975, 1774, 1698, 1520, 1432, 1380, 1251, 1150, 1073, 955, 775, $741 \mathrm{~cm}^{-1} ;{ }^{1} \mathrm{H}$ NMR (400 MHz, DMSO): $\delta 10.97$ (bs, $1 \mathrm{H}, \mathrm{NH}$ ), 8.40$8.38(\mathrm{~m}, 1 \mathrm{H}, \mathrm{Ar}-\mathrm{H}), 7.75-7.67$ (m, 2H, Ar-H), 7.47-6.99 (m, 6H, Ar$\mathrm{H}), 4.78(\mathrm{dd}, 1 \mathrm{H}, J=4.74 \mathrm{~Hz}, 3.34 \mathrm{~Hz}, 2-\mathrm{H}), 4.07(\mathrm{~d}, 1 \mathrm{H}, J=5.36 \mathrm{~Hz}$, $\mathrm{NH}), 3.79$ (dd, $1 \mathrm{H}, J=3.01 \mathrm{~Hz}, 2.76 \mathrm{~Hz}, 3-\mathrm{H}$ ), 3.57 (s, $3 \mathrm{H}, \mathrm{OCH}_{3}$ ), 3.47 (d, $1 \mathrm{H}, J=2.64 \mathrm{~Hz}, 4-\mathrm{H}$ ), 3.39 (d, 1H, J=14.08 Hz, 6-H), 3.15 (d, $\left.1 \mathrm{H}, J=14.08 \mathrm{~Hz}, 6-\mathrm{H}^{\prime}\right), 2.33\left(\mathrm{~s}, 3 \mathrm{H}, \mathrm{NCH}_{3}\right) \mathrm{ppm} ;{ }^{13} \mathrm{C} \mathrm{NMR}(100$ MHz, DMSO): $\delta 179.5(\mathrm{C}=0), 176.1(\mathrm{C}=0), 172.5(\mathrm{C}=0), 159.9$, $148.5,136.6,135.8,127.4,124.6,122.3,121.0,120.9,118.6$, 118.1, 111.5, 107.6, 72.1, 66.7, 52.2, 51.7, 50.8, 29.5, $24.1 \mathrm{ppm}$; MS (ESI, M+H'): m/z $419.2\left(\mathrm{M}+\mathrm{H}^{+}, 100\right)$. HRMS (DIP): m/z [M+] calculated for $\mathrm{C}_{23} \mathrm{H}_{22} \mathrm{~N}_{4} \mathrm{O}_{4}$, 418.1641; found: 418.1649 .

Methyl(2S,3S,6aR)-6a-((1H-indol-3-yl)methyl)-2-(3chlorophenyl)-4,6-dioxo-5-phenyloctahydropyrrolo[3,4b]pyrrole-3-carboxylate (5ge): After $36 \mathrm{~h}$ and work up the product ( $503 \mathrm{mg}, 98 \%$ yield) was isolated and crystallised as colourless prisms: $\operatorname{mp} 169-171{ }^{\circ} \mathrm{C}$; IR $v_{\max }$ : 3315, 3060, 2983, 
2950, 1782, 1739, 1703, 1595, 1436, 1392, 1253, 1240, 1164, 981, $747 \mathrm{~cm}^{-1}$; ${ }^{1} \mathrm{H}$ NMR (400 MHz, DMSO): $\delta 11.08$ (bs, $1 \mathrm{H}, \mathrm{NH}$ ), 7.75 (d, 1H, J= 7.80 Hz, Ar-H), 7.53-6.64 (m, 13H, Ar-H), 4.78 (dd, $1 \mathrm{H}, J=5.42 \mathrm{~Hz}, 5.42 \mathrm{~Hz}, 2-\mathrm{H}), 4.24(\mathrm{~d}, 1 \mathrm{H}, J=5.48 \mathrm{~Hz}, \mathrm{NH}), 3.72$ (d, $1 \mathrm{H}, J=4.44 \mathrm{~Hz}, 4-\mathrm{H}), 3.58\left(\mathrm{~s}, 3 \mathrm{H}, \mathrm{OCH}_{3}\right), 3.55$ (d, $1 \mathrm{H}, J=14.12$ $\mathrm{Hz}, 6-\mathrm{H}$ ), 3.43 (dd, $1 \mathrm{H}, J=5.42 \mathrm{~Hz}, 4.48 \mathrm{~Hz}, 3-\mathrm{H}), 3.30$ (d, 1H, $J=$ $\left.14.18 \mathrm{~Hz}, 6-\mathrm{H}^{\prime}\right) \mathrm{ppm} ;{ }^{13} \mathrm{C}$ NMR (100 MHz, DMSO): $\delta 179.1$ (C=0), $176.3(\mathrm{C}=0), 173.1$ ( $\mathrm{C}=0), 145.3,137.5,134.8,133.3,131.0,129.4$ ( $2 \times$ C), 129.1, 128.7, 128.4, 127.5 ( $2 \times$ C), 127.4, 126.0, 125.8, $122.5,120.0,119.6,112.4,109.3,72.7,66.6,55.9,52.8,52.5,30.5$. MS (ESI, M-H $\left.\mathrm{H}^{+}\right): \mathrm{m} / \mathrm{z} 512.2\left(\mathrm{M}+\mathrm{H}^{+}, 100\right), 514.2\left(\mathrm{M}+\mathrm{H}^{+}, 35\right)$. HRMS (DIP): $\mathrm{m} / \mathrm{z}\left[\mathrm{M}^{+}\right]$calculated for $\mathrm{C}_{29} \mathrm{H}_{24} \mathrm{ClN}_{3} \mathrm{O}_{4}, 513.1455$; found: 513.1450 .

\section{Methyl(2S,3S,6aR)-6a-((1H-indol-3-yl)methyl)-2-(3-} chlorophenyl)-5-methyl-4,6-dioxooctahydropyrrolo[3,4b]pyrrole-3-carboxylate (5gf): After $26 \mathrm{~h}$ and work up the product ( $429 \mathrm{mg}, 95 \%$ yield) was isolated and crystallised as colourless prisms: $\mathrm{mp} 152-154{ }^{\circ} \mathrm{C}$; IR $v_{\max }$ : $3344,3270,3060$, $2982,2949,1780,1737,1705,1378,1288,1173,747,681 \mathrm{~cm}^{-1}$; ${ }^{1} \mathrm{H}$ NMR (400 MHz, DMSO): $\delta 11.00(\mathrm{bs}, 1 \mathrm{H}, \mathrm{NH}), 7.70(\mathrm{~d}, 1 \mathrm{H}, J=$ $7.76 \mathrm{~Hz}, \mathrm{Ar}-\mathrm{H}), 7.40-7.02(\mathrm{~m}, 8 \mathrm{H}, \mathrm{Ar}-\mathrm{H}), 4.66(\mathrm{dd}, 1 \mathrm{H}, J=5.68 \mathrm{~Hz}$, $5.68 \mathrm{~Hz}, 2-\mathrm{H}$ ), 4.06 (d, $1 \mathrm{H}, J=5.28 \mathrm{~Hz}, \mathrm{NH}), 3.54\left(\mathrm{~s}, 3 \mathrm{H}, \mathrm{OCH}_{3}\right), 3.50$ (d, $1 \mathrm{H}, J=4.88 \mathrm{~Hz}, 4-\mathrm{H}$ ), 3.43 (d, $1 \mathrm{H}, J=14.20 \mathrm{~Hz}, 6-\mathrm{H}$ ), 3.22 (d, $\left.1 \mathrm{H}, J=14.20 \mathrm{~Hz}, 6-\mathrm{H}^{\prime}\right)$ ), 3.13 (dd, $1 \mathrm{H}, J=6.36 \mathrm{~Hz}, 4.96 \mathrm{~Hz}, 3-\mathrm{H}$ ), 2.59 (s, 3H, $\left.\mathrm{NCH}_{3}\right)$ ppm; ${ }^{13} \mathrm{C}$ NMR (100 MHz, DMSO): $\delta 178.8$ $(\mathrm{C}=0), 176.1(\mathrm{C}=0), 171.5(\mathrm{C}=0), 143.9,135.9,132.9,130.0$, $127.4,127.3,126.2,125.2,124.7,121.0,118.6,118.2,111.5$, 107.6, 70.9, 65.3, 54.1, 52.1, 51.1, 28.8, 24.1 ppm; MS (ESI, M+H+ $\mathrm{m} / \mathrm{z} 452.2\left(\mathrm{M}+\mathrm{H}^{+}, 100\right), 454.2\left(\mathrm{M}+\mathrm{H}^{+}, 35\right)$. HRMS (DIP): $\mathrm{m} / \mathrm{z}\left[\mathrm{M}^{+}\right]$ calculated for $\mathrm{C}_{24} \mathrm{H}_{22} \mathrm{ClN}_{3} \mathrm{O}_{4}$, 451.1299; found: 451.1301 .

Methyl (2S,3S,3aS,6aR)-6a-((1H-indol-3-yl)methyl)-1(benzoylcarbamothioyl)-4,6-dioxo-2,5-

diphenyloctahydropyrrolo[3,4-b]pyrrole-3-carboxylate

(6ga): After $24 \mathrm{~h}$ and work up the product (552 mg, $86 \%$ yield) was isolated and crystallised as pale yellow prisms: $\mathrm{mp} 150-152$ ${ }^{\circ} \mathrm{C}$; IR $v_{\max }$ : 3202, 3060, 2981, 2889, 1787, 1739, 1702, 1537, $1492,1389,1252,1202,923,743,704 \mathrm{~cm}^{-1} ;{ }^{1} \mathrm{H}$ NMR (400 MHz, DMSO): $\delta 11.60$ (bs, $1 \mathrm{H}, \mathrm{NH}), 11.33$ (bs, $1 \mathrm{H}, \mathrm{NH}), 8.11$ (d, $2 \mathrm{H}, J=$ $7.40 \mathrm{~Hz}, \mathrm{Ar}-\mathrm{H}$ ), 7.72-7.00 (m, 17H, Ar-H), 6.80 (bs, 1H, 2-H), 4.22 (d, $1 \mathrm{H}, J=1.44 \mathrm{~Hz}, 4-\mathrm{H}$ ), 3.91 (brs, $2 \mathrm{H}, 6-\mathrm{H}$ ve $6-\mathrm{H}^{\prime}$ ), 3.46 (dd, $1 \mathrm{H}$, $J=1.76 \mathrm{~Hz}, 1.76 \mathrm{~Hz} 3-\mathrm{H}), 2.78\left(\mathrm{~s}, 3 \mathrm{H}, \mathrm{OCH}_{3}\right) \mathrm{ppm} ;{ }^{13} \mathrm{C}$ NMR $(100$ MHz, DMSO): $\delta 179.7(\mathrm{C}=\mathrm{S}), 176.4(\mathrm{C}=0), 173.9(\mathrm{C}=0), 168.7$ $(\mathrm{C}=0), 165.1(\mathrm{C}=0), 138.5,135.4,133.3,133.0,130.8,129.2$, 129.6 ( $2 \times \mathrm{C}), 129.0(2 \times \mathrm{C}), 128.8$ ( $2 \times \mathrm{C}), 127.8$ ( $2 \times \mathrm{C}), 127.8$, 127.3, 126.5 ( $2 \times \mathrm{C}), 126.2,125.1$ ( $2 \times \mathrm{C}), 121.1,118.8,118.1$, 111.4, 104.5, 74.2, 69.2, 54.2, 51.5, 50.1, 26.7 ppm; MS (ESI, $\left.\mathrm{M}+\mathrm{H}^{+}\right): \mathrm{m} / \mathrm{z} 643.2\left(\mathrm{M}+\mathrm{H}^{+}, 100\right)$; HRMS (DIP): $\mathrm{m} / \mathrm{z}\left[\mathrm{M}^{+}\right]$calculated for $\mathrm{C}_{37} \mathrm{H}_{30} \mathrm{~N}_{4} \mathrm{O}_{5} \mathrm{~S}$, 642.1937; found: 642.1930.

Methyl (2S,3S,3aS,6aR)-6a-((1H-indol-3-yl)methyl)-1(benzoylcarbamothioyl)-5-methyl-4,6-dioxo-2-

phenyloctahydropyrrolo[3,4-b]pyrrole-3-carboxylate

(6gb): After $24 \mathrm{~h}$ and work up the product (516 mg, $89 \%$ yield) was isolated and crystallised as pale yellow prisms: mp 207-209 o $C$; IR $v_{\max }$ : 3267, 3187, 3060, 3027, 2885, 1786, 1741, 1686, 1546, 1445, 1225, 955, $703 \mathrm{~cm}^{-1}$; ${ }^{1} \mathrm{H}$ NMR (400 MHz, DMSO): $\delta$ 11.74 (bs, $1 \mathrm{H}, \mathrm{NH}), 11.25$ (bs, $1 \mathrm{H}, \mathrm{NH}), 8.12$ (d, $2 \mathrm{H}, J=7.24 \mathrm{~Hz}$, Ar-H), 7.73-7.59 (m, 4H, Ar-H), 7.38-6.98 (m, 9H, Ar-H), 6.64 (brs, $1 \mathrm{H}, 2-\mathrm{H}), 3.97$ (d, 1H, J = 1.84 Hz, 4-H), 3.76 (brs, 2H, 6-H ve 6-H'), 3.24 (dd, $1 \mathrm{H}, J=2.16 \mathrm{~Hz}, 2.16 \mathrm{~Hz}, 3-\mathrm{H}$ ), 2.81 (s, 3H, OCH $H_{3}$, 2.75 (s, 3H, $\left.\mathrm{NCH}_{3}\right)$ ppm; ${ }^{13} \mathrm{C}$ NMR (100 MHz, DMSO): $\delta 178.8(\mathrm{C}=\mathrm{S})$, 178.0 (C=0), 174.6 ( $\mathrm{C}=0), 168.7$ ( $\mathrm{C}=0), 164.9$ ( $\mathrm{C}=0), 138.7,135.4$, 133.3, 133.1, 129.1 ( $2 \times \mathrm{C}), 128.6(2 \times \mathrm{C}), 127.8(2 \times \mathrm{C}), 127.7$, $127.2,126.0,124.8$ ( $2 \times \mathrm{C}), 121.1,118.8,118.0,111.4,104.574 .2$, 69.2, 53.9, 51.5, 50.0, 26.2, 25.3 ppm; MS (ESI, M+H+): m/z 580.6 $\left(\mathrm{M}+\mathrm{H}^{+}, 100\right)$; HRMS (DIP): $\mathrm{m} / \mathrm{z}\left[\mathrm{M}^{+}\right]$calculated for $\mathrm{C}_{32} \mathrm{H}_{28} \mathrm{~N}_{4} \mathrm{O}_{5} \mathrm{~S}$, 580.1780; found: 580.1776 .

Methyl (2S,3S,3aS,6aR)-6a-((1H-indol-3-yl)methyl)-1(benzoylcarbamothioyl)-4,6-dioxo-5-phenyl-2-(pyridin-2-

yl)octahydropyrrolo[3,4-b]pyrrole-3-carboxylate (6gc): After $30 \mathrm{~h}$ and work up the product ( $489 \mathrm{mg}, 76 \%$ yield) was isolated and crystallised as pale yellow prisms: mp 170-172 ${ }^{\circ} \mathrm{C}$; IR $v_{\max }$ : 3357, 2981, 1782, 1755, 1738, 1698, 1538, 1255, 1238, 743,706 cm ${ }^{-1}$; ${ }^{1} \mathrm{H}$ NMR (400 MHz, DMSO): $\delta 11.92$ (bs, $1 \mathrm{H}, \mathrm{NH}$ ), 11.33 (bs, $1 \mathrm{H}, \mathrm{NH}), 8.45-8.43$ (m, 1H, Ar-H), 8.01-7.06 (m, 18H, Ar-H), 6.56 (brs, 1H, 2-H), 4.25 (s, 1H, 4-H), 3.90 (d, 1H, $J=15.06$ $\mathrm{Hz}, 6-\mathrm{H}$ ), 3.84 (d, 1H, J = $\left.15.12 \mathrm{~Hz}, 6-\mathrm{H}^{\prime}\right), 3.24$ (s, 1H, 3-H), 2.73 (s, $3 \mathrm{H}, \mathrm{OCH}_{3}$ ) ppm; ${ }^{13} \mathrm{C}$ NMR (100 MHz, DMSO): $\delta 176.6(\mathrm{C}=\mathrm{S}), 176.5$ $(\mathrm{C}=0), 173.2(\mathrm{C}=0), 167.5(\mathrm{C}=0), 163.6(\mathrm{C}=0), 156.8,148.2$, $135.7,134.2,132.0,131.9,130.2,128.1$ ( $2 \times \mathrm{C}), 128.1,128.0(2 \mathrm{x}$ C), 126.6, $126.4(2 \times \mathrm{C}), 125.4(2 \times \mathrm{C}), 124.9,122.8,121.9,120.0$, 117.6, 117.1, 110.2, 103.4, 73.0, 69.5, 53.4, 50.4, 46.4, 25.8 ppm; MS (ESI, M+H'): m/z $644.2\left(\mathrm{M}+\mathrm{H}^{+}, 100\right) ; H R M S$ (DIP): $\mathrm{m} / \mathrm{z}$ [M+] calculated for $\mathrm{C}_{36} \mathrm{H}_{29} \mathrm{~N}_{5} \mathrm{O}_{5} \mathrm{~S}, 643.1889$; found: 643.1883 .

Methyl (2S,3S,3aS,6aR)-6a-((1H-indol-3-yl)methyl)-1(benzoylcarbamothioyl)-5-methyl-4,6-dioxo-2-(pyridin-2-

yl)octahydropyrrolo[3,4-b]pyrrole-3-carboxylate (6gd): After $24 \mathrm{~h}$ and work up the product ( $453 \mathrm{mg}, 78 \%$ yield) was isolated and crystallised as pale yellow prisms: $\mathrm{mp}$ 198-200 ${ }^{\circ} \mathrm{C}$; IR $v_{\max }: 3170,3060,2961,1785,1755,1738,1685,1553,1449$, $1357,1233,1007,748,705 \mathrm{~cm}^{-1}$; ${ }^{1} \mathrm{H}$ NMR (400 MHz, DMSO): $\delta$ 11.99 (bs, 1H, NH), 11.25 (bs, 1H, NH), 8.41-8.39 (m, 1H, Ar-H), 8.02-8.00 (m, 2H, Ar-H), 7.76-7.60 (m, 5H, Ar-H), 7.37-7.00 (m, $6 \mathrm{H}, \mathrm{Ar}-\mathrm{H}), 6.56(\mathrm{~d}, 1 \mathrm{H}, J=1.24 \mathrm{~Hz} 2-\mathrm{H}), 3.99(\mathrm{~d}, 1 \mathrm{H}, J=1.36 \mathrm{~Hz}, 4-$ $\mathrm{H}), 3.35$ (brs, $2 \mathrm{H}, 6-\mathrm{H}$ ve 6-H'), 3.07 (s, $\left.3 \mathrm{H}, \mathrm{OCH}_{3}\right), 3.07-3.05(\mathrm{~m}$, 1H, 3-H), 2.65 (s, 3H, NCH$)_{3}$ ppm; ${ }^{13} \mathrm{C}$ NMR (100 MHz, DMSO): $\delta$ $178.9(\mathrm{C}=\mathrm{S}), 177.5(\mathrm{C}=0), 175.3(\mathrm{C}=0), 168.7(\mathrm{C}=0), 164.7(\mathrm{C}=0)$, 157.8, 149.4, 136.7, 135.4, 133.2, 133.1, 129.1 ( $2 \times \mathrm{C}), 127.7$, 127.6 ( 2 x C), 125.9, 123.6, 122.9, 121.0, 118.8, 118.0, 111.3, 104.7, 74.1, 70.4, 54.5, 51.5, 47.5, 26.5, 25.5. MS (ESI, M+H+): m/z $581.6\left(\mathrm{M}+\mathrm{H}^{+}, 100\right)$; HRMS (DIP): $\mathrm{m} / \mathrm{z}\left[\mathrm{M}^{+}\right]$calculated for $\mathrm{C}_{31} \mathrm{H}_{27} \mathrm{~N}_{5} \mathrm{O}_{5} \mathrm{~S}$, 581.1733; found: 581.1727.

Methyl (2S,3S,3aS,6aR)-6a-((1H-indol-3-yl)methyl)-1(benzoylcarbamothioyl)-2-(3-chlorophenyl)-4,6-dioxo-5phenyloctahydropyrrolo[3,4-b]pyrrole-3-carboxylate (6ge): After $24 \mathrm{~h}$ and work up the product (554 mg, $82 \%$ yield) was isolated and crystallised as pale yellow prisms: mp 157-159 ${ }^{\circ} \mathrm{C}$; IR $v_{\max }$ : 3387, 3196, 3051, 2956, 1787, 1704, 1529, 1491, 1348, 1255, 1191, 755, $699 \mathrm{~cm}^{-1}$; ${ }^{1} \mathrm{H}$ NMR (400 MHz, DMSO): $\delta 11.51$ (bs, 1H, NH), 11.31 (bs, 1H, NH), 8.09 (d, 2H, $J=7.24 \mathrm{~Hz}, \mathrm{Ar}-\mathrm{H}$ ), 7.71-7.03 (m, 17H, Ar-H), 6.69 (bs, 1H, 2-H), 4.22 (d, 1H, $J=1.84$ $\mathrm{Hz}, 4-\mathrm{H}$ ), 3.91 (brs, 2H, 6-H ve 6-H'), 3.44 (dd, 1H, J=2.30 Hz, 2.30 $\mathrm{Hz} 3-\mathrm{H}), 2.76$ (s, 3H, OCH3) ppm; ${ }^{13} \mathrm{C}$ NMR (100 MHz, DMSO): $\delta$ 180.5 (C=S), 175.5 ( $\mathrm{C}=0), 173.8(\mathrm{C}=0), 168.5(\mathrm{C}=0), 165.4(\mathrm{C}=0)$, $141.5,135.5,133.5,133.3,132.9,130.9,130.6,129.2,129.1(2 \mathrm{x}$ C), $128.9(2 \times$ C), $127.9(2 \times$ C), 127.8, 127.4, 126.4 ( $2 \times$ C), 126.1, 125.2, 124.0, 121.1, 118.8, 118.1, 111.4, 104.5, 74.7, 69.2, 54.1, 51.6, 49.9, 26.7 ppm; MS (ESI $\left.\mathrm{M}+\mathrm{H}^{+}\right): \mathrm{m} / \mathrm{z} 678.2\left(\mathrm{M}+\mathrm{H}^{+}, 100\right)$, 
$679.2\left(\mathrm{M}+\mathrm{H}^{+}, 35\right)$; HRMS (DIP): $\mathrm{m} / \mathrm{z}\left[\mathrm{M}^{+}\right]$calculated for $\mathrm{C}_{37} \mathrm{H}_{29} \mathrm{ClN}_{4} \mathrm{O}_{5} \mathrm{~S}, 676.1547$; found: 676.1544 .

Methyl(2S,3S,3aS,6aR)-6a-((1H-indol-3-yl)methyl)-1(benzoylcarbamothioyl)-2-(3-chlorophenyl)-5-methyl-4,6dioxooctahydropyrrolo[3,4-b]pyrrole-3-carboxylate (6gf): After $24 \mathrm{~h}$ and work up the product (538 $\mathrm{mg}, 84 \%$ ) was isolated and crystallised as pale yellow prisms: mp 192-194 ํㅜ; IR $v_{\max }$ 3384, 3203, 2982, 2951, 1784, 1745, 1693, 1537, 1365, 1352, $1254,1237,1213,752,693 \mathrm{~cm}^{-1} ;{ }^{1} \mathrm{H}$ NMR (400 MHz, DMSO): $\delta$ 11.67 (bs, 1H, NH), 11.24 (bs, 1H, NH), 8.10 (d, 2H, $J=7.40 \mathrm{~Hz}, \mathrm{Ar}-$ H), 7.73-7.56 (m, 4H, Ar-H), 7.37-7.23 (m, 4H, Ar-H), 7.11-6.95 (m, 4H, Ar-H), 6.53 (bs, 1H, 2-H), 3.96 (d, 1H, $J=1.88 \mathrm{~Hz}, 4-\mathrm{H}$ ), 3.75 (brs, $2 \mathrm{H}, 6-\mathrm{H}$ ve 6-H'), 3.26 (dd, $1 \mathrm{H}, J=2.36 \mathrm{~Hz}, 2.36 \mathrm{~Hz} 3-\mathrm{H}$ ), $2.84\left(\mathrm{~s}, 3 \mathrm{H}, \mathrm{OCH}_{3}\right), 2.74\left(\mathrm{~s}, 3 \mathrm{H}, \mathrm{NCH}_{3}\right) \mathrm{ppm} ;{ }^{13} \mathrm{C} \mathrm{NMR}(100 \mathrm{MHz}$ DMSO): $\delta 179.2(\mathrm{C}=\mathrm{S}), 177.5(\mathrm{C}=0), 174.5(\mathrm{C}=0), 168.5(\mathrm{C}=0)$, $165.0(\mathrm{C}=0), 141.4,135.4,133.4,133.2,133.1,130.5,129.0(2 \mathrm{x}$ C), 127.8 ( $2 \times$ C), 127.6, 127.3, 125.9, 125.2, 123.5, 121.1, 118.8, 117.9, 111.4, 104.5, 74.5, 69.0, 53.8, 51.6, 49.7, 26.3, 25.3 ppm; MS (ESI, M+H+ $\left.\mathrm{H}^{+}\right): \mathrm{m} / \mathrm{z} 615.2\left(\mathrm{M}+\mathrm{H}^{+}, 100\right), 616.1\left(\mathrm{M}+\mathrm{H}^{+}, 35\right)$; HRMS (DIP): $\mathrm{m} / \mathrm{z}$ [M+] calculated for $\mathrm{C}_{32} \mathrm{H}_{27} \mathrm{ClN}_{4} \mathrm{O}_{5} \mathrm{~S}, 614.1391$; found: 614.1386 .

\section{General Procedure Compound 7gg}

To a stirred solution of bicyclic pyrrolidine $4 \mathrm{gg}$ (0,4 g, 0,85 mmol) in $\mathrm{MeOH}$ (not anhydrous, $20 \mathrm{~mL}$ ) was added dropwise a solution of sodium methoxide $(0,38 \mathrm{~g}, 2,04 \mathrm{mmol})$ in dry $\mathrm{MeOH}(10 \mathrm{~mL})$ over $10 \mathrm{~min}$ and the mixture stirred and heated at reflux temperature for $36 \mathrm{~h}$. The solvent was evaporated under reduced pressure, quenched with saturated aqueous ammonium chloride then extracted thrice with $\mathrm{CH}_{2} \mathrm{Cl}_{2}$. The combined organic solvents were dried over $\mathrm{MgSO}_{4}$ and filtered. The product $\mathbf{7 g g}(0,13 \mathrm{~g}$, 95\%) crystallised from $\mathrm{CH}_{2} \mathrm{Cl}_{2}$ as colourless solid. Mp 207-209 ${ }^{\circ} \mathrm{C}$ (dec).

(2R,3R,3aR,6aS)-6a-((1H-indol-3-yl)methyl)-2-(4chlorophenyl)-5-ethyl-4,6-dioxo octahydropyrrolo[3,4-

\section{Notes and references}

(1) Patil, M. M.; Rajput, S. S. Int. J. Pharm. Pharm. Sci. 2014, 6, 8-14.

(2) (a) Kumar, S.; Prakash, S.; Gupta, K.; Dongre, A.; Balaram, P.; Balaram, H. Nat. Commun. 2016, 7, 1-14.

(3) For other applications of maleimides, see: (a) Miller, C. W.; Jönsson, E. S.; Hoyle, C. E.; Viswanathan, K.; Valente, E. J. J. Phys. Chem. B 2001, 105, 2707-2717; (b) Dolci, E. Froidevaux, V.; Joly-Duhamel, C.; Auvergne, R.; Boutevin, B.; Caillol, S. Polymers 2016, 56, 512-556.

(4) Wang, L.; Ni, Q.; Blümel, M.; Shu, T.; Raabe, G.; Enders, D. Chem. Eur. J. 2015, 21, 1-6.

(5) (a) Dondas, H. A.; Retamosa, M. de G.; Sansano, J. M. Synthesis 2017, 49, 2819-2851; (b) Wróbel, M. Z.; Chodkowski, A.; Herold, F.; Gomólka, A.; Kleps, J.; Mazurek, A. P.; Plucinski, F.; Mazurek, A.; Nowak, G.; Siwek, A.; Stachowicz, K.; Slawinska, A.; Wolak, M.; Szewczyk, B.; Satala, G.; Bojarski, A. J.; Turlo, J. Eur. J. Med. Chem. 2013, 63, 484-500; (c) Gupta, P.; Garg, P.; Roy, N. Med. Chem. Res. 2010, 22, 5014-5028; (d) Nájera, C.; Sansano, J. M. Curr. Top. Med. Chem. 2014, 14, b]pyrrole-3-carboxylic acid (7gg): The product 7gg (130 mg, $95 \%$ yield) was isolated and crystallised from $\mathrm{CH}_{2} \mathrm{Cl}_{2}$ as colourless solid: mp: $207-209{ }^{\circ} \mathrm{C}(\mathrm{dec})$; IR $\nu_{\max }$ : 3429, 3304, 3065, 2979, 2934, 2905, 2831, 1770 ( $\mathrm{C}=0), 1724(\mathrm{C}=0), 1675(\mathrm{C}=0)$, $831 \mathrm{~cm}^{-1}$; ${ }^{1} \mathrm{H}$ NMR (400 MHz, DMSO): $\delta 12.8$ (brs, $1 \mathrm{H}, \mathrm{OH}$ ), 10.98 (br s, 1H, NH), 7.70 (d, 1H, J= $7.88 \mathrm{~Hz}, \operatorname{Ar} H$ ) , 7.42-7.27 (m, 5H, $\operatorname{Ar} H$ ) , 7.11-6.96 (m, 3H, ArH), $4.72(\mathrm{~d}, 1 \mathrm{H}, J=6.52 \mathrm{~Hz}, 5 H), 3.49$ (d, $1 \mathrm{H}, J=5 \mathrm{~Hz}, 3 H), 3.44(\mathrm{~s}, 1 \mathrm{H}, \mathrm{NH}), 3.4(\mathrm{~d}, 1 \mathrm{H}, J=14 \mathrm{~Hz}, 6 H), 3.22(\mathrm{~d}$, $\left.1 \mathrm{H}, J=13.92 \mathrm{~Hz}, 6^{\prime} H\right), 3.15-3.04\left(\mathrm{~m}, 2 \mathrm{H}, \mathrm{CH}_{2} \mathrm{CH}_{3}\right), 2.99$ (dd, $1 \mathrm{H}, J=$ $5.16 \mathrm{~Hz}, J=6.46 \mathrm{~Hz}, 4 \mathrm{H}), 0.62\left(\mathrm{t}, 3 \mathrm{H}, J=7.16 \mathrm{~Hz}, \mathrm{CH}_{2} \mathrm{C}_{3}\right) \mathrm{ppm} ;{ }^{13} \mathrm{C}$ NMR (100 MHz, DMSO): $\delta 178.7(\mathrm{C}=0), 176.1(\mathrm{C}=0), 173.0(\mathrm{C}=0)$, 140.6, 135.8, 131.8, 128.3 ( 2 x C), 128.0 ( $2 \times$ C), 127.3, 124.4, 121.0, 118.6, 118.3, 111.4, 107.7, 71.0, 65.6, 54.7, 51.1, 32.7, 29.1, 11.8 ppm; MS (ESI, M+H+): m/z $452.2\left(\mathrm{M}^{+}, 100, \mathrm{Cl}: 35\right) / 454.2$ $\left(\mathrm{M}^{+}, 33.3, \mathrm{Cl}: 37\right)-(3 / 1)$ and $453.2(\mathrm{M}+1,100, \mathrm{Cl}: 35) / 455.2(\mathrm{M}+1$, 33.3, Cl: 37) -(3/1); HRMS (DIP): $\mathrm{m} / \mathrm{z}\left[\mathrm{M}^{+}\right]$calculated for $\mathrm{C}_{24} \mathrm{H}_{22} \mathrm{ClN}_{3} \mathrm{O}_{4}, 451.1299$; found: 451.1289 .

\section{Acknowledgments}

We are grateful for support from Mersin University (Project no: MEU-2017-COL-01007-M150D and BAP-SBE AKB (SB) 2012-8 YL and BAP 2015- AP2-1342. We gratefully acknowledge financial support from the Spanish Ministerio de Economía y Competitividad (MINECO) (projects CTQ2013-43446-P and CTQ2014-51912-REDC), the Spanish Ministerio de Economía, Industria y Competitividad, Agencia Estatal de Investigación (AEI) and Fondo Europeo de Desarrollo Regional (FEDER, EU) (projects CTQ2016-76782-P and CTQ2016-81797-REDC), the Generalitat Valenciana (PROMETEOII/2014/017), the Gobierno Vasco/Eusko Jaurlaritza (GV/EJ, grant IT673-13), and the University of Alicante. O.L. gratefully acknowledges UPV/EHU for her postdoctoral grant. O.L. and A.d.C. gratefully thanked SGI/IZO-SGIker and DIPC for generous allocation of computational resources. 
1105-1150; (e) Nájera, C.; Sansano, J. M. Org. Biomol. Chem. 2009, 7, 4567-4581.

(6) Nural, Y.; Döndas, H. A.; Grigg, R.; Sahin, E. Heterocycles 2011, 83, 2091-2114.

(7) For previous contributions of our group in the study of pharmaceutical properties of new compounds, see: $(a)$ Poyraz, S.; Belveren, S.; Ulger, M.; Sahin, E.; Dondas, H. A. Monatsh Chem 2017, 148, 2173-2182; (b) Poyraz, S.; Canacankatan, N.; Belveren, S.; Yetkin D.; Kibar, K.; Ülger, M.; Sansano, J. M.; Özcelik, N. D.; Yilmaz, S. N.; Döndaş, H. A. Monatsh. Chem. 2018, accepted.

(8) Belveren, S.; Döndas, H. A.; Ülger, M.; Poyraz, S.; GarcíaMingüens, E.; Ferrandiz-Saperas, M.; Sansano, J. M. Tetrahedron 2017, 73, 6718-6727.

(9) (a) Wellington, K.; Plosker, G. L. Drugs 2002, 62, 15391574; (b) Zhang, M. Z.; Chen, Q.; Yang, G. F. Eur. J. Med. Chem. 2015, 89, 421-441; (c) Sherer, C.; Snape, T. J. Eur. J. Med. Chem. 2015, 97, 552-560; (d) Zhang, M. Z.; Mulholland, N.; Beattie, D.; Irwin, D.; Gu, Y. C.; Chen, Q.; Yang, G. F.; Clough, J. Eur. J. Med. Chem. 2013, 63, 22-32; (e) Leneva, I. A.; Russel, R. J.; Boriskin, Y. S.; Ha, A. J. Antiviral Res. 2009, 81, 132-140; (f) Kaushik, N. K.; Kaushik, N.; Attri, P.; Kumar, N.; Kim, C. H.; Verma, A. K.; Choi, E. H. Molecules 2013, 18, 6620-6662; (g) Welsch, M. E.; Syner, S. A.; Stockwell, B. R. Curr. Op. Chem. Biol. 2010, 14, 347-361.

(10) Compound 5ga was obtained previously by our group in $85 \%$ yield, see ref. 6

(11) CCDC-1534206 for compound 7gg contains supplementary crystallographic data for the structure. Copies of these data can be obtained free of charge on application to CCDC, 12 Union Road, Cambridge CB2 1EZ, UK; FAX: (+44) 1223 336033, or online via www.ccdc.cam.ac.uk/data_request/cif, or by emailing data request@ccdc.cam.ac.uk.

(12) $\beta$-Proline derivatives exhibit anticancer or antibacterial activities: (a) Kudryavtsev, K. V.; Yu, C.-C.; Ivantcova, P. M.; Polshakov, V. I.; Churakov, A. V.; Braese, S.; Zefirov, N. S.; Guh, J. H. Chem. Asian J. 2015, 10, 383-389; (b) Ferrazzano, L.; Viola, A.; Lonati, E.; Bulbarelli, A.;
Musumeci, R.; Cocuzza, C.; Lombardo, M.; Tolomelli, A. Eur. J. Med. Chem. 2016, 124, 906-919; (c) Fjelbye, K.; Marigo, M.; Clausen, R. P.; Juhl, K.; Karsten, J. Synlett 2017, 28, 231-234.

(13) CCDC-1533867† for compound $\mathbf{6 g f}$ contains supplementary crystallographic data for the structure. Copies of these data can be obtained free of charge on application to CCDC, 12 Union Road, Cambridge CB2 1EZ, UK; FAX: (+44) 1223 336033, or online via www.ccdc.cam.ac.uk/data_request/cif, or by emailing data_request@ccdc.cam.ac.uk

(14) (a) Palomino, J. C.; Portaels, F. Eur. J. Clin. Microbiol. Infect. Dis. 1999, 18, 380-383; (b) National Committee for Clinical Laboratory Standards. Susceptibility Testing of Mycobacteria, Nocardia, and Other Aerobic Actinomycetes: Approved Standard NCCLS Document M24-a. NCCLS. 2003 (Wayne, Pennsylvania).

(15) National Committee for Clinical Laboratory Standards. Tentativ Standard- Second Edition NCCLS Document M24-T. Susceptibilitiy Testing of Mycobacteria, Nocardia and other aerobic Actinomycetes. 2002. Pennsylvania USA).

(16) Grigg R, Gunaratne H. Q.N.; Sridharan V. Tetrahedron 1987, 43, 5887-5898.

(17) Dondas H. A.; O. Altinbas, Heterocycl. Commun. 2004, 10, 167-172.

(18) Becke, A. D. J. Chem. Phys. 1993, 98, 5648-5650.

(19) Gaussian09, Revision E.01, Frisch M. J. et al., Gaussian Inc., Wallingford CT, 2009 (full reference in the Supporting Information).

(20) Parr, R. G.; Yang, W. Density-Functional Theory of Atoms and Molecules, Oxford, New York, 1989.

(21) Grimme, S.; Antony, J.; Ehrlich, S.; Krieg, H. J. Chem. Phys. 2010, 132, 154104.

(22) Cammi, R.; Mennucci, B.; Tomasi, J.J. Phys. Chem. A 2000, 104, 5631-5637.

(23) Tomasi, J.; Mennucci, B.; Cammi, R. Chem. Rev. 2005, 105, 2999-3093. 